\title{
Versuche über die Abhängigkeit der Atmung von Arenicola marina (Annelides Polychaeta) von Größe und Temperatur
}

\author{
FRIEDRICH KRÜGER \\ Biologische Anstalt Helgoland, Zentrale, Hamburg-Altona
}

\begin{abstract}
Experiments concerning the dependence of respiration of Arenicola marina (Annelides Polychaeta) on size and temperature. A knowledge of the rate of oxygen consumption is very important for the evaluation of many physiological and ecological problems. Among the many factors affecting respiratory rate, water temperature and body size are particularly considered here. The modifying effects of body size may be expressed mathematically by the allometric formula: $y=b \cdot w^{a}$, where $b$ represents the rate of $O_{2}$ consumption of an individual whose weight is expressed in a chosen metrical weight unity (i. e. in grams, ounces, etc.), and $\alpha$ represents the decrease of metabolic rate during growth. In Arenicola the exponent is not the same at all temperatures tested. In most cases it lies between 0.7 and 0.8 , i. e., between a weight proportional respiratory rate and a surface proportional one. Minimum values for $\alpha$ were found in experiments conducted in summer at $20^{\circ} \mathrm{C}$ and in spring at $15^{\circ} \mathrm{C}$. They characterize an optimum efficiency of metabolism at these temperatures. Determinations of $\mathrm{b}$ demonstrated that metabolic rate of Arenicola is significantly less affected in spring $\left(10^{\circ}\right.$ to $\left.20^{\circ} \mathrm{C}\right)$ and autumn $\left(10^{\circ}\right.$ to $\left.25^{\circ} \mathrm{C}\right)$ than is usually known from biological processes. However, the temperature coefficients above and below these temperature ranges are very high. Another break in the temperature-rate curve could be demonstrated below $5^{\circ} \mathrm{C}$ in spring.
\end{abstract}

\section{EINLEITUNG}

Die Stoffumsetzungen im Organismus hängen von einer Reihe Faktoren ab, von denen Temperatur, Tiergröße, Tätigkeitszustand, Jahres- und Tageszeit hier erwähnt seien. Die vollständige Analyse des Stoffwechsels eines Tieres hat also eine große Zahl von Faktoren zu berücksichtigen. Erst wenn einmal die Wirkung aller dieser Faktoren auf den Stoffumsatz erfaßt ist, kann man einen Aufschluß über die physiologische Einpassung eines Organismus in seine Umgebung erwarten. Von diesem Ziel sind wir zur Zeit aber noch sehr weit entfernt. Die Klärung aller dieser Fragen wird dadurch erschwert, daß zwischen den einzelnen Faktoren Wechselwirkungen bestehen und wir sie daher nicht getrennt voneinander behandeln können. Die Temperaturabhängigkeit des Stoffwechsels scheint $z$. B. von der Tiergröße beeinflußt $z u$ werden, andererseits ist $z u$ erwarten, daß sie auch jahreszeitlich variiert. Das Problem der physiologischen Einpassung erweist sich also als außerordentlich vielschichtig. So kann jeder Beitrag nur 
einen kleinen Baustein liefern, und auch die vorliegenden Untersuchungen können die aufgeworfenen Fragen nur in begrenzten Ausschnitten beantworten.

Den Kernpunkt vorliegender Messungen bildete das Problem der Größenabhängigkeit der Atmung, deren Kenntnis für die Auswertung anderer Versuchsreihen erforderlich war. Schon 1958 war ich auf diese Frage eingegangen und hatte aus den damaligen Messungen auf eine nahezu gewichtsproportionale Atmung geschlossen. In der $Z$ wischenzeit hat sich aber gezeigt, daß der Sauerstoffverbrauch von Arenicola merklich von einer Gewichtsproportionalität abweicht. Daher habe ich diese Frage erneut mit einer etwas anderen Versuchstechnik aufgegriffen. Bei den neuen Messungen, die zunächst speziell auf dieses Problem ausgerichtet waren, kamen Würmer mit sehr viel stärker differierendem Gewicht zur Verwendung als in den früheren Versuchen. Bei der unvermeidbaren Schwankungsbreite der Einzelwerte bei Atmungsmessungen kann man nur dann zu zahlenmäßig zuverlässigen Ergebnissen kommen, wenn man Tiere sehr unterschiedlicher Größe in den Versuchen benutzt, so daß die Atmungsdifferenz die individuellen Schwankungen erheblich überschreitet.

Die Untersuchungen der drei letzten Jahrzehnte haben gezeigt, daß bei der Mehrzahl der Tiere - einschließlich der Evertebraten - im Verlauf des Wachstums der Quotient Sauerstoffverbrauch : Gewicht, oder anders ausgedrückt, der Sauerstoffverbrauch je Gewichtseinheit, abnimmt. An sich ist dieses Problem nicht mehr von so akutem Interesse, praktisch ist es aber eminent wichtig; denn es zwingt uns, in anderer Weise als bislang allgemein üblich, die Atmungsgröße eines Tieres so zu charakterisieren, daß die Größenfunktion erkennbar wird. Der mathematische Weg hierzu ist in der allometrischen Formel gegeben:

$$
\begin{aligned}
y & =\mathrm{b} \cdot \mathrm{w}^{a} \\
(y=\text { Stoffwechselgröße; } \mathrm{w} & =\text { Gewicht } \alpha \text { und } \mathrm{b}=\text { Konstanten })
\end{aligned}
$$

Bei der Darstellung in einem doppelt logarithmischen Koordinatensystem liegen im Idealfall die Versuchswerte als Funktion von w auf einer Geraden. Der tg des Steigungswinkels dieser Geraden ist der Exponent $\alpha$, dessen Wert im allgemeinen $z$ wischen 0,66 und 1,00 liegt.

Die Stoffwechselreduktion im Verlaufe des Wachstums ist ein Phänomen, das man schon im Beginn des vorigen Jahrhunderts erkannte und das man in eine mathematische Form zu fassen sich bemühte. Einen ersten Versuch unternahm RaveAux (1837/39) zitiert nach v. Hoessurn (1888) -, der die Wärmeabgabe von Säugetieren als der Oberflächenentwicklung parallel verlaufend ansetzte:

$$
\begin{gathered}
\mathrm{W}=\mathrm{a} \cdot \mathrm{K}^{2 / 3} \\
(\mathrm{~W}=\text { Wärmebildung; } \mathrm{K}=\text { Körpergewicht } ; \mathrm{a}=\text { Konstante })
\end{gathered}
$$

In der Formel von Rameaux gibt der Exponent $2 / 3$ die Oberflächenabhängigkeit des Stoffwechsels wieder, die besagt, daß im Verlauf des Wachstums, bezogen auf die Gewichtseinheit, eine Stoffwechselreduktion stattfindet. Kausal deutete man diese Stoffwechselreduktion dadurch, daß zur Erhaltung der normalen Körpertemperatur der homoiothermen Tiere ein gewisser Stoffwechsel erforderlich sei, der den Wärme- 
verlust an der Oberfläche ausgleicht. Aus diesem Zusammenhang glaubte man die Oberflächenparallelität des Stoffwechsels erklären zu können. Nun hat sich in neuerer Zeit aber erstens gezeigt, daß auch bei Warmblütlern die vermutete strenge Proportionalität zur Oberfläche nicht gegeben ist, sondern der Exponent der Formel von RAMEAUX näher bei 3/4 liegt und was noch wesentlicher ist, daß zweitens die „Oberflächenregel ${ }^{\star}$ auch für eine Reihe von wechselwarmen Tieren zutriff.

Aus der Zeit der Diskussion um die Gültigkeit oder Nidhtgültigkeit der Oberflächenregel scheint noch die Geptlogenheit vieler Autoren zu stammen, aus ihren Versuchszahlen nur den Exponenten $\alpha$ der allometrischen Gleichung zu berechnen und anzugeben. Der Exponent $\alpha$, der die Größenfunktion wiedergibt, ist zwar wichtig, aber isoliert für sich, ohne erheblichen Aussagewert, er scheint auch für die einzelne Art keine konstante Größe zu sein und sich in Abhängigkeit von der Temperatur zu ändern. Uberdies variiert $a$ auch beim interspezifischen Vergleich in nur relativ engen Grenzen, die bislang noch keinerlei Beziehungen zu physiologischen oder biologischen Gegebenheiten erkennen lassen.

Der Faktor $\mathrm{b}$ der allometrischen Gleichung ist die Atmungsgröße eines Tieres von der Gewichtseinheit, berechnet aus der Gesamtheit der Versuchswerte. Im Gegensatz zur einfachen Durchschnittsberechnung aus dem arithmetischen Mittel der Versuchswerte schließt $\mathrm{b}$ die Größenfunktion ein. b spricht auf alle Faktoren an, von denen die Atmungsgröße abhängt und ist dadurch der einwandfreie marhematische Weg zur Darstellung ihrer Wirkung. Mit der Kenntnis von $\alpha$ und $\mathrm{b}$ ist außerdem die Möglichkeit gegeben, die Atmungsgröße von Tieren beliebiger anderer Gewichte zu berechnen.

Unabhängig von jeder Deutung wird somit die allometrische Formel ein wesentliches und unentbehrliches Werkzeug der Stoffwechselphysiologie. Das bedingt aber, daß wir uns bei irgendwelchen Messungen nicht mehr des gewichtsbezogenen Durchschnittes einiger Versuchswerte bedienen dürfen, sondern genügend Zahlenmaterial sammeln müssen, um die Konstanten $\alpha$ und $\mathrm{b}$ der allometrischen Gleichung mit hinreichender Sicherheit zu berechnen.

Die zusätzliche Komplikation, der wir hierbei in vielen Fällen gegenüberstehen, ist die, daß sich anscheinend nicht nur jahreszeitliche Unterschiede in der Atmungsgröße finden, sondern daß auch die Temperaturfunktion ihre Gestalt im Laufe des Jahres ändert. Da Einzelmessungen keine eindeutige Aussage ermöglichen, wird ersichtlich, welch riesige Zahl von Versuchen prinzipiell erforderlich wäre, um die Atmungsphysiologie eines Organismus in ihrer Abhängigkeit von nur drei Faktoren zu charakterisieren.

\section{VERSUCHSBESCHREIBUNG}

Die Atmungsmessungen erfolgten ausschließlich in den Laboratorien auf Helgoland und an Wurmmaterial, das vom Ostufer der Insel stammte. Dieser Hinweis erscheint wichtig, da Material von List auf Sylt aus noch nicht übersehbaren Gründen teilweise abweichende Werte lieferte. Die Versuchstiere befanden sich vor und zwischen den Versuchen in Käfigen ohne Sand mit Seewasserdurchfluß. Die Würmer hielten sich unter diesen Bedingungen sehr gut 1 bis 2 Wochen. Die Temperatur des Seewassers lag im Frühjahr bei etwa $10^{\circ} \mathrm{C}$, im Herbst bei etwa $18^{\circ} \mathrm{C}$. 
Bei den früheren Untersuchungen hatte ich die Würmer zur Messung in U-förmigen Rohren untergebracht, um möglichst natürliche Bedingungen im Experiment zu schaffen. In den jetzigen Versuchen nahmen wir von diesen U-Rohren Abstand, denn die Würmer waren nicht nur in diesen Rohren sehr unruhig, unterbrachen dabei durch Einkriechen in die Seewasserzuleitungen die Wasserzufuhr und machten auf diese Weise zahlreiche Messungen zunichte, sondern ihre Pumptätigkeit war auch in den Rohren durch lange Pausen unterbrochen. Da die aktive Pumptätigkeit der Würmer Voraussetzung für die Wirkungsweise der Atmungsmessungen in diesen Rohren war, ergab sich hiermit auch die Ursache für die in den früheren Messungen beobachtete außerordentlich starke Schwankung der Atmungsgröße.

Bei den jetzigen Versuchen kam die Apparatur, die ich für die Atmungsmessung an den Miesmuscheln entwickelt hatte, unverändert zur Verwendung. In der Mehrzahl der Versuche verhielten sich die Würmer in den zylindrischen Tierbehältern recht ruhig. Die in diesem Apparat erhaltenen Werte, der dazu noch den Vorteil einer einfacheren Handhabung aufweist, zeigten eine wesentlich bessere Konstanz. Größenordnungsmäßig stimmten sie mit den früheren Untersuchungen überein.

$\mathrm{Zu}$ den immer wieder $\mathrm{zu}$ beobachtenden starken Unregelmäßigkeiten in der Atmungsgröße von Arenicola möchte ich bemerken, daß sie zum Teil vielleicht ihre Ursache in Verletzungen haben, die die Würmer beim Ausgraben aus dem Sand erleiden. Nach meinen Beobachtungen im Verlauf von anderen Untersuchungen kommt es hierdurch sehr häufig zu Gefäßzerreißungen und Darmverletzungen, in Verbindung hiermit dürften auch Quetschungen des Hautmuskelschlauches zu erwarten sein. Es ist sicher, daß diese äußerlich gar nicht erkennbaren Schädigungen ihre Rückwirkung auf die Körperfunktion der Würmer haben.

In der Versuchsreihe im Frühjahr 1962 stülpten wir über die Tierbehälter passende Messing-Kappen, um die Würmer vor dem einfallenden Tageslicht abzuschirmen. Ich vermag noch nicht eindeutig zu sagen, in welcher Weise sich diese Maßnahme auswirkt.

Da die Mehrzahl der Versuche bei Temperaturen unter der Raumtemperatur erfolgte, benutzten wir zur Temperaturregelung das Prinzip der gesteuerten Kühlung (KRÜGER 1957). In einem Kühlgerät wurde das als Thermostaten-Flüssigkeit dienende Leitungswasser abgekïhlt und konnte durch eine Pumpe in den Thermostaten geworfen werden, in dem sich die Atmungsapparatur befand. Der Thermostat besaß einen Uberlauf, durch den das überschüssige Wasser wieder in den Wasserbehälter des Kühlgerätes zurückfloß und dort erneut gekühlt wurde. Die Pumpe war durch ein Kontaktthermometer über ein Relais gesteuert, das in dem Augenblick, wo die Temperatur in dem Thermostatenbad die eingestellte Temperatur überschritt, die Pumpe so lange in Tätigkeit setzte, bis durch den Zustrom kalten Wassers die Soll-Temperatur erreicht war.

Das Verfahren der gesteuerten Kühlung gestattete die rationellste Ausnutzung der Kälte-Erzeugung des Kühlgerätes, die noch unterstützt wurde durch eine gute Wärmeisolierung des Thermostatenbades und der Schlauchleitungen. So war es möglich, auch bei sommerlich hohen Raumtemperaturen im Thermostatenbad Temperaturen in der Nähe des Gefrierpunktes aufrechtzuerhalten. Hierbei ist zu beachten, daß das Kühlaggregat zusätzlich noch das durch die Atmungsapparatur strömende Wasser 
auf die Versuchstemperatur abkühlen muß. Die Schwankungen um den Soll-Wert betrugen nur wenige hundertstel Grade. Bei den Versuchen, bei denen Temperaturen über der Umgebungstemperatur erforderlich waren, wurde das Thermostatenbad durch einen Aquarienheizer von etwa 100 Watt Stromaufnahme über die eingestellte Temperatur kontinuierlich aufgeheizt und die Regulation wieder durch den gesteuerten Kühlwasserzufluß bewirkt. Die Einstellung des gesamten Temperaturbereiches erforderte also praktisch keinerlei Anderungen an der Apparatur. Um möglichst hohe Temperaturkonstanz zu erreichen, empfiehlt es sich, die Temperatur in dem Kühlaggregat immer nur wenige Grade tiefer einzustellen, als die Temperatur im Thermostatenbad betragen soll.

Bei den Versuchen über den Temperatureinfluß arbeiteten wir im allgemeinen an einem Tag mit zwei verschiedenen Temperaturen, um die einzelnen Versuchsserien nicht allzu sehr in die Länge ziehen zu müssen. Zur Anpassung der Versuchstiere an die Versuchstemperatur begannen wir stets erst $1^{1 / 2}$ bis 2 Stunden nach dem Erreichen der neuen Temperatur mit den Messungen. Hierdurch ergaben sich relativ lange Pausen zwischen den einzelnen Temperaturserien, so daß in ihnen meist nur 4 Messungen von je 30 Minuten Dauer durchgeführt werden konnten.

Bei den übrigen Versuchen konnten den Berechnungen Mittelwerte aus 5-6 und mehr Messungen zugrunde gelegt werden. Die Auswertung der Versuche erfolgte wie oben ausgeführt - auf Grund der allometrischen Formel (1). Der Exponent $\alpha$ berechnet sich nach der Formel:

$$
a=\frac{\Sigma \log x \cdot \log y}{2 \log x^{2}-\frac{\Sigma \log x \cdot \Sigma \log y}{N}}
$$

Die Konstante $b$ ergibt sich zu:

$$
\log \mathrm{b}=\frac{\Sigma \log \mathrm{y}-\mathrm{b} \cdot \Sigma \log \mathrm{x}}{\mathrm{N}}
$$

( $a=$ Tangens des Steigungswinkels; $\mathrm{x}=$ Wurmgewicht; $\mathrm{y}=$ Sauerstoffverbrauch; $\mathrm{N}=$ Anzahl der Wertpaare.)

Für die praktische Durchführung der Berechnungen sei z. B. auf meine Arbeit von 1960 verwiesen.

\section{DIE GROSSENABHANGIGKEIT DER ATMUNG BEI $15^{\circ} \mathrm{C}$}

Es ist, wie oben ausgeführt, nicht zulässig, die Atmungsgröße eines Tieres einfach als Quotienten aus Sauerstoffverbrauch und Gewicht, also bezogen auf die Gewichtseinheit, anzugeben, auch wenn dieses Verfahren heute noch vielfach geübt wird. Eine eindeutige Kennzeichnung ist nur nach der allometrischen Formel (1) durch Angabe der darin enthaltenen Parameter a und b möglich.

Angesichts der unvermeidlichen starken Streuung der Einzelwerte bei Atmungsmessungen, die sich auch nicht vollständig vermeiden läß̊t, wenn man Mittelwerte aus einer großen Anzahl von Versuchswerten bildet, lassen sich mathematische Auswertungen nur dann mit Aussicht auf Erfolg durchführen, wenn man die stark diffe- 
rierende Atmungsgröße von Tieren mit sehr verschiedenem Gewicht erfaßt. Unter dieser Bedingung tritt die durch das Objekt bedingte Unsicherheit der Meßwerte zurïck gegenüber der im Prinzip zugrunde liegenden Differenz, die dann auch die zugehörige mathematische Funktion erkennen läßt.

Aus diesem Grunde ist es erforderlich, Tiere von möglichst unterschiedlichem Gewicht zur Untersuchung heranzuziehen. Als Mindestverhälttis der großen zu den kleinen Individuen wird 1:10 angegeben, eine Spanne, die in der Hauptversuchsserie bei $15^{\circ} \mathrm{C}$ vom Herbst 1960 bei weitem überschritten wurde. Wir erfaßten hierbei Würmer mit Gewichten zwischen 0,104 und $14,17 \mathrm{~g}$; das Gewichtsverhältnis betrug also $1: 136^{1}$.

Tabelle 1

Sauerstoff verbrauch von Arenicola marina, Messungen im Aug./Sept. 1960

\begin{tabular}{|c|c|c|c|c|c|}
\hline $\begin{array}{l}\text { Gewicht } \\
\text { in } g\end{array}$ & $\begin{array}{c}\text { O2-Verbrauch } \\
\text { in } \mathrm{ml} / \mathrm{h}\end{array}$ & $\begin{array}{c}\mathrm{O}_{2}-\text { Verbrauch } \\
\text { in } \mathrm{ml} / \mathrm{g} / \mathrm{h}\end{array}$ & $\begin{array}{l}\text { Gewicht } \\
\text { in } g\end{array}$ & $\begin{array}{l}\mathrm{O}_{2}-V_{\text {Verbrauch }} \\
\text { in } \mathrm{ml} / \mathrm{h}\end{array}$ & $\begin{array}{l}\mathrm{O}_{2-V e r b r a u c h} \\
\text { in } \mathrm{ml} / \mathrm{g} / \mathrm{h}\end{array}$ \\
\hline 0,104 & 14,00 & 134,62 & 4,25 & 191,00 & 44,94 \\
\hline 0,113 & 8,93 & 79,03 & 4,35 & 196,57 & 45,19 \\
\hline 0,148 & 13,33 & 90,07 & 4,55 & 234,20 & 51,47 \\
\hline 0,159 & 12,78 & 80,38 & 4,83 & 199,40 & 41,28 \\
\hline 0,255 & 30,18 & 118,35 & 5,01 & 267,88 & 53,47 \\
\hline 0,300 & 23,43 & 78,10 & 5,25 & 172,00 & 32,76 \\
\hline 0,311 & 22,92 & 73,70 & 5,90 & 238,80 & 40,47 \\
\hline 0,498 & 36,00 & 72,29 & 6,00 & 248,20 & 41,37 \\
\hline 0,896 & 96,22 & 77,25 & 6,46 & 350,33 & 54,23 \\
\hline 1,33 & 61,08 & 45,92 & $6 ; 54$ & 257,00 & 39,30 \\
\hline 1,91 & 107,91 & 56,50 & 6,61 & 251,20 & 38,00 \\
\hline 2,13 & 131,00 & 61,50 & 6,74 & 245,67 & 36,45 \\
\hline 2,52 & 130,20 & 51,67 & 6,75 & 239,58 & 35,49 \\
\hline 2,60 & 174,00 & 66,92 & 7,03 & 269,00 & 38,26 \\
\hline 2,80 & 136,71 & 48,83 & 7,05 & 283,63 & 40,23 \\
\hline 3,14 & 120,00 & 38,22 & 7,10 & 271,60 & 38,25 \\
\hline 3,16 & 211,83 & 67,03 & 7,15 & 363,13 & 50,79 \\
\hline 3,25 & 148,80 & 45.78 & 7,21 & 304,00 & 42,16 \\
\hline 3,27 & 168,75 & 51,61 & 7,86 & 282,80 & 35,98 \\
\hline 3,29 & 163,20 & 49,60 & 8,08 & 308,18 & 38,14 \\
\hline 3,31 & 124,25 & 37,54 & 8,13 & 350,00 & 43,05 \\
\hline 3,32 & 106,17 & 31,98 & 10,59 & 437,33 & 41,30 \\
\hline 3,38 & 154,83 & 45,81 & 11,00 & 293,20 & 26,65 \\
\hline 4,00 & 118,57 & 29,64 & 12,64 & 370,67 & 29,33 \\
\hline 4,04 & 196,40 & 48,61 & 14,17 & 640,00 & 45,17 \\
\hline
\end{tabular}

Wir arbeiteten in dieser Versuchsreihe bei $15^{\circ} \mathrm{C}$, weil die Würmer diese Temperatur anscheinend gut während des ganzen Jahres ertragen. Daher war es die Standardtemperatur, die auch in anderen Versuchen zur Anwendung kam. Die breite Gewichtsspanne der Versuche vom August und September 1960 (Tab. 1) gestattete

1 Noch größere Würmer standen uns damals nicht zur Verfügung. Während bei Würmern aus dem Watt von List/Sylt das von uns registrierte Maximalgewicht eines Wurmes $26,5 \mathrm{~g}$ beträgt, war das Maximalgewicht eines Helgoländer Wurmes $18,8 \mathrm{~g}$. Dabei bereitet die Beschaffung so großer Würmer auf Helgoland Schwierigkeiten, während sie in List jederzeit erhältlich sind. Es bliebe zu klären, ob dieser Unterschied durch die Ernährungs- oder Temperaturverhältnisse bedingt ist oder ob verschiedene Rassen vorliegen. 
eine sehr zuverlässige Bestimmung der Parameter der Gleichung (1). Es ergab sich aus ihnen für die Größe b - also den Sauerstoffverbrauch eines Wurmes von $1 \mathrm{~g}$ Gewicht $62,01 \mathrm{cmm} / \mathrm{h}$ und für den Exponenten $\alpha$ der Wert 0,77 .

Zur Frage der Reduzierbarkeit dieser Werte seien die Zahlen gebracht, die im folgenden Jahr (Tab. 2) aus einer kleineren Gewichtsspanne - zwischen 0,38 und $13,3 \mathrm{~g}-$ und wesentlich weniger Messungen gewonnen wurden: $\mathrm{b}=59,4 \mathrm{cmm} / \mathrm{h}$ und $\alpha=0,75$. Angesichts der großen Streuung der zugrunde liegenden Werte muß man das Ergebnis beider Versuchsreihen als identisch betrachten.

Wie aus Abbildung 1 hervorgeht, verteilen sich die Meßpunkte im ganzen Bereich ziemlich gleichmäßig auf beiden Seiten der Ausgleichsgeraden. Hieraus darf man

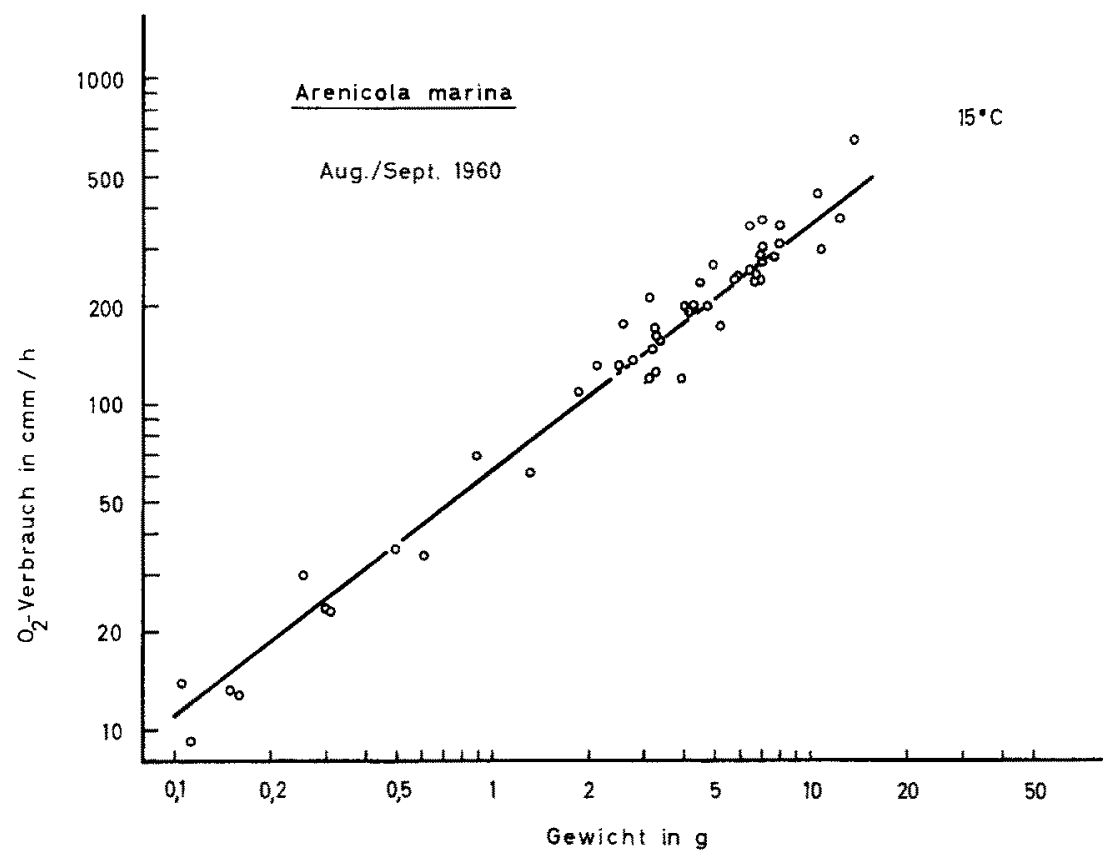

Abb. 1: Größenabhängigkeit der Atmung von Arenicola bei $15^{\circ} \mathrm{C}$. Versuche vom Herbst 1960. Darstellung im doppelt logarithmischen Koordinatensystem, berechnete Regressions-Gerade eingezeichnet

schließen, daß bei der Streuung der Werte Meßfehler nur eine untergeordnete Rolle spielen. Wären die Abweichungen von der Ausgleichsgeraden durch methodische Fehler bedingt, so müßte man bei den niederen Sauerstoffverbrauchswerten eine größere Streuung finden als bei den höheren Verbrauchswerten, da im logarithmischen Koordinatensystem schon kleine numerische Abweichungen bei den niederen Werten starke Verlagerung der Punkte zur Folge haben. Es dürfte damit bei den Messungen die Forderung erfüllt gewesen sein, daß im ganzen erfaßten Bereich die Meßgenauigkeit identisch war. Es bleibr somit der Schlu\}, daß die beobachteten Abweichungen von dem mathematischen Mittelwert eine wirklich gegebene Variabilität der individuellen Atmungsgröße wiedergeben. 
Die Messungen im Warburg-Apparat, die nur eine sehr viel engere Gewichtsspanne - zwischen 0,07 und $0,61 \mathrm{~g}$ - umfassen, lieferten für $\mathrm{b}$ nahezu den gleichen Wert von $60,3 \mathrm{cmm} / \mathrm{h}$, für $\alpha$ aber einen höheren Wert, nämlich 0,91 . Dieser Unterschied im Regressionskoeffizienten besagt, daß die Atmungswerte in der WarburgApparatur etwas niedriger liegen als bei der chemischen Sauerstoffmessung.

$\mathrm{Da}$ die Möglichkeit bestand, daß bei letzterer die Atmungsmessung an so kleinen Objekten versagt, wurden die Werte für $\alpha$ und b einmal für die Würmer unter $1,5 \mathrm{~g}$ und zum anderen über 1,5 g getrennt berechnet. Der Wert für $\alpha$ war mit 0,76 für beide Gruppen identisch, eine unwesentliche Differenz ergab sich für $b$, das für die kleinen Würmer den Wert $60,7 \mathrm{cmm} / \mathrm{h}$ hat, für die großen Würmer $63,1 \mathrm{cmm} / \mathrm{h}$. Die Zusammenfassung der gesamten Versuche mit der chemischen Sauerstoffbestimmung erscheint daher gerechtfertigt.

Aus welchem Grunde die Versuche im Warburg-Apparat nur größenordnungsmäßig vergleichbare Werte ergaben, ist nicht klar ersichtlich. Man kann daran denken, $\mathrm{daß}$ die recht intensive Schüttelbewegung für die Atmung abweichende Verhältnisse schuf. Wenn auch das Gesamtbild nicht wesentlich verschoben würde, ist es aber aus diesem Grunde nicht möglich, die mit der Warburg-Technik erhaltenen Zahlen zusammen mit den übrigen Meßwerten zu verarbeiten.

Die Sommerversuche hatten somit zu gut übereinstimmenden Werten mit verschiedener Methodik und in verschiedenen Jahren geführt. Leider kann man nicht das gleiche von den Versuchen im Frühjahr 1961 und 1962 sagen (Tab. 3 u. 4). Hierbei dürfte der Umstand eine Rolle spielen, daß es in beiden Versuchsserien nicht möglich war, ganz kleine Würmer unter $1 \mathrm{~g}$ Gewicht für die Versuche zu crhalten; denn gerade diese kleinsten Tiere sind für eine zuverlässige Berechnung des Exponenten $\alpha$ außerordentlich wichtig. Die Parameter der Gleichung (1) konnten also nur aus einer wesentlich kleineren Gewichtsspanne berechnet werden; sie betrug in beiden Versuchsreihen nur etwa 1 : 8. Wenn alle Bemühungen, kleinste Würmer im Frühjahr zu beschaffen, erfolglos waren, so dürtte die Ursache darin zu suchen sein, daß es sich in den Sommerversuchen um einjährige Tiere handelte, die im Jahr zuvor mit ihrer Entwicklung begonnen hatten und die im Frühjahr noch zu klein waren, um beim Graben am Ufer gefunden zu werden. Diese jüngsten Stadien sind ja erst in letzter Zeit entdeckt worden (Newell 1948, 1949). Zu dem Fehlen der kleinsten Würmer kommt hinzu, daß nur ein wesentlich kleineres Zahlenmaterial zur Verfügung stand, da die Versuche bei $15^{\circ} \mathrm{C}$ im Rahmen von Temperaturversuchen gewonnen wurden. Trotzdem seien die erhaltenen Zahlen hier aufgeführt, die auch in den Tabellen 3 und 4 in anderem Zusammenhang enthalten sind.

Für b ergaben die Versuche von 1961 einen Wert von $70,7 \mathrm{cmm} / \mathrm{h}$, der also nennenswert höher liegt als die Sommerzahlen. Im Frühjahr 1962 fand sich dagegen ein Wert von $63,1 \mathrm{cmm} / \mathrm{h}$, der in der Größenordnung der Sommerversuche liegt. Da in diesen Versuchen aber erstmalig mit verdunkelten Tierbehältern gearbeitet wurde, könnte der geringere Wert dadurch bedingt sein, daß die Tiere sich bei diesen Messungen durch den Fortfall des Lichtreizes ruhiger verhielten. Auffällig ist ferner, daß in beiden Versuchsreihen der Wert des Exponenten niedriger lag als im Sommer. Er betrug im Frühjahr 1961 nur 0,72 und 1962 sogar nur 0,67, wie er für eine oberflächenproportionale Atmung gültig ist. Die gleichsinnige Anderung des Exponenten 
in den beiden Frühjahrs-Serien läßt trotz der Bedenken bezüglich der unbedingten Zuverlässigkeit der gefundenen Zahlen die Möglichkeit einer jahreszeitlichen Änderung der Größenfunktion erkennen.

Beiläufig wurde auch in einer Versuchsserie über 24 Stunden, die im Verlaufe eines Kurses von Studenten der Universität Hamburg durchgeführt wurde, die Frage der tageszeitlichen Schwankungen der Atmungsgröße geprüft, wie sie in neverer Zeit z. B. bei Krebsen (BRown, BenNeT \& Webb 1954) nachgewiesen wurden. Die Messungen im Warburg-Apparat ergaben eine geringe Erhöhung der Atmungswerte um Mitternacht, die aber bei den Messungen in der Durchströmungsapparatur nicht hervortrat. $\mathrm{Da}$ alle Versuche dieser Arbeit etwa zur gleichen Tageszeit liefen, war es für die vorliegende Fragestellung nicht erforderlich, tageszeitliche Schwankungen in Betracht $z u$ ziehen.

Tabelle 2

Atmungswerte von Arenicola marina. Messungen im Sept./Okt. 1961, Helgoland. Durchschnittlicher Sauerstoffverbrauch in $\mathrm{cmm} / \mathrm{h}$

\begin{tabular}{|ccccccccc|}
\hline $\begin{array}{c}\text { Gewicht } \\
\text { in g }\end{array}$ & $2^{0} \mathrm{C}$ & $3^{0} \mathrm{C}$ & $5^{0} \mathrm{C}$ & $10^{\circ} \mathrm{C}$ & $15^{\circ} \mathrm{C}$ & $20^{\circ} \mathrm{C}$ & $25^{\circ} \mathrm{C}$ & $30^{\circ} \mathrm{C}$ \\
\hline 0,38 & 10,60 & 12,35 & 13,50 & 21,25 & 26,00 & 35,00 & 18,10 & 72,75 \\
0,48 & - & - & 10,75 & - & 37,25 & 31,50 & - & $-\overline{-}$ \\
0,69 & - & - & 15,68 & 41,50 & 57,43 & 54,00 & - & - \\
0,86 & - & - & - & - & 69,50 & 87,75 & - & - \\
0,87 & - & 13,50 & - & 35,25 & 40,50 & 50,00 & 62,50 & 100,75 \\
1,03 & 21,25 & 18,25 & 36,25 & 35,75 & 46,00 & 62,33 & 61,25 & 89,75 \\
1,32 & 18,50 & 21,00 & 31,25 & 35,75 & 52,75 & 74,67 & 86,75 & 127,25 \\
1,53 & 17,25 & 21,00 & 31,75 & 83,75 & 77,80 & 91,00 & 110,25 & 132,75 \\
1,75 & 9,50 & 60,00 & 34,75 & 98,33 & 108,00 & 116,50 & 130,25 & - \\
1,82 & - & 45,75 & - & - & - & - & - & 169,67 \\
2,24 & - & - & - & - & 106,60 & - & - & - \\
2,54 & 32,00 & - & 56,67 & 139,33 & 134,33 & 141,00 & 242,00 & - \\
2,89 & 24,25 & 30,00 & 35,75 & 41,17 & 99,00 & 131,50 & 170,25 & 277,00 \\
3,29 & - & - & 88,25 & 153,75 & 166,20 & 232,25 & 224,75 & 311,00 \\
3,37 & - & - & 70,50 & 137,25 & 198,83 & 216,33 & - & 317,75 \\
3,39 & - & - & 83,75 & - & 198,75 & 251,33 & - & 367,25 \\
4,23 & 31,00 & 48,00 & 63,00 & 119,50 & 190,50 & 144,75 & 189,50 & 260,25 \\
4,95 & 56,00 & 60,50 & 145,50 & 128,50 & 236,50 & 247,50 & 379,25 & 565,25 \\
5,00 & - & - & - & 87,00 & 121,33 & - & - & - \\
5,97 & 48,00 & 44,00 & 76,00 & 115,83 & 210,75 & 222,50 & 334,50 & 503,75 \\
6,37 & - & - & 80,75 & 173,00 & 290,57 & 304,67 & - & 530,75 \\
6,70 & 43,00 & 83,75 & 89,25 & 179,00 & 192,60 & 174,00 & 275,75 & 381,25 \\
8,09 & - & - & - & - & - & 437,25 & 520,50 & - \\
8,19 & - & - & - & - & - & - & - & 542,80 \\
9,08 & - & - & 174,25 & 335,50 & 398,14 & 394,00 & - & 567,50 \\
10,31 & 126,60 & 156,75 & 182,75 & 282,00 & 351,60 & 384,50 & 517,75 & - \\
10,68 & 140,00 & 149,75 & 248,50 & 397,00 & 437,43 & 585,75 & 633,25 & 914,50 \\
11,30 & 140,40 & 164,25 & 163,75 & 292,00 & 394,40 & 386,25 & 512,75 & - \\
11,96 & 161,00 & 164,67 & 225,25 & 323,75 & 341,50 & 565,00 & 618,00 & 689,00 \\
12,64 & - & - & - & - & - & - & - & 838,60 \\
12,71 & 145,25 & 171,25 & 151,50 & 350,75 & 329,40 & - & - & - \\
13,16 & 164,75 & 128,75 & 167,25 & 322,00 & 306,00 & 204,00 & 633,75 & - \\
13,29 & 203,00 & 215,50 & 235,00 & 483,00 & 448,57 & 274,50 & 327,00 & - \\
\hline
\end{tabular}


Bei der unterirdischen Lebensweise der Würmer dürfte physiologisch der Tag- und Nachtwechsel von untergeordneter Bedeutung sein. Näherliegend wäre eine den Gezeiten folgende Periodik, für die wir bislang noch keinen Hinweis gefunden haben, die aber in gesonderten Versuchen gegebenenfalls geprüft werden müßte.

\section{DER SAUERSTOFFVERBRAUCH VON ARENICOLA IN SEINER ABHANGIGKEIT VON DER TEMPERATUR}

Der Größenabhängigkeit des Stoffwechsels kommt keine direkt erkennbare ökologische Bedeutung zu. Ihre Kenntnis ist aber für die Auswertung der Stoffwechselversuche grundlegend. Demgegenüber ist die Temperaturwirkung ein Problem, das nicht nur vom Standpunkt des Physiologen gesehen sehr widhtig ist, sondern ebensosehr den Okologen interessiert. Die Temperaturwirkung auf die Vorgänge im Organismus läuft der Temperaturwirkung auf unbelebte Systeme in dem Sinne parallel, daß im allgemeinen auch die Stoffumsetzungen im Organismus zunehmen. Das gilt aber - wie ge-

Tabelle 3

Atmungswerte von Arenicola marina. Messungen im Mai/Juni 1961, Helgoland.

Durchschnittlicher Sauerstoff verbrauch in $\mathrm{cmm} / \mathrm{h}$

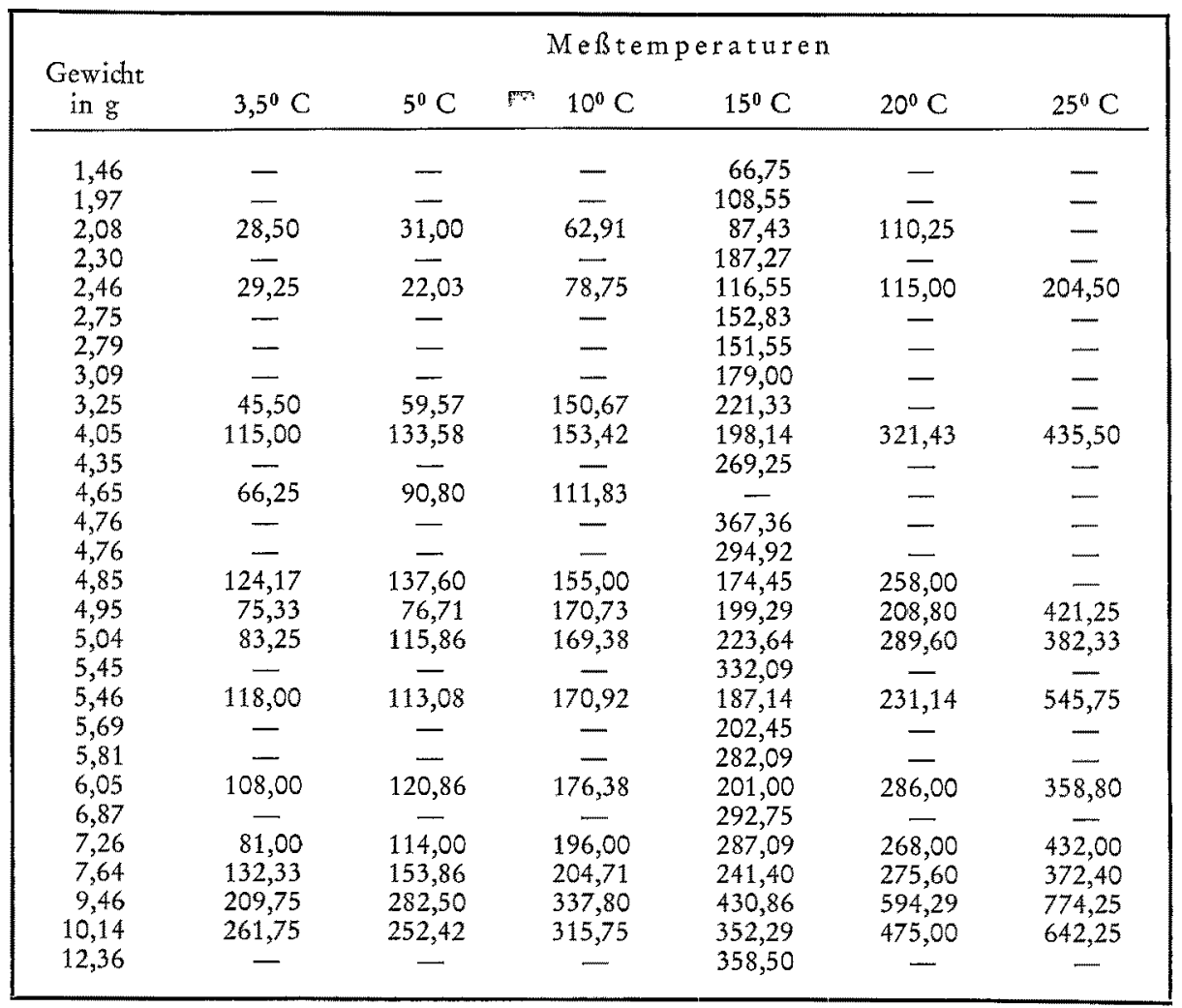




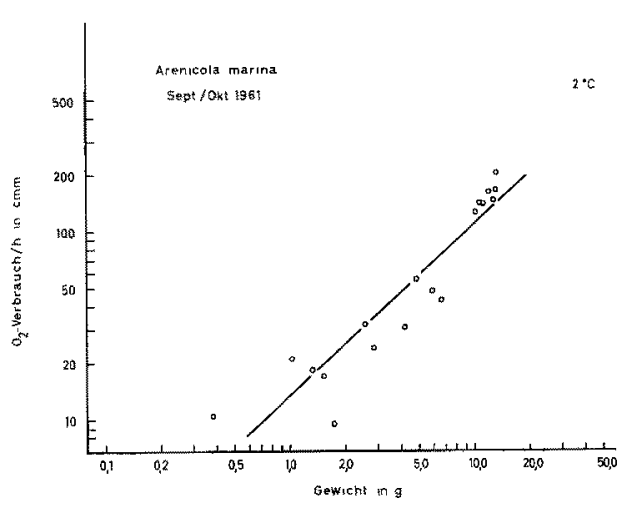

Abb. $2 a$

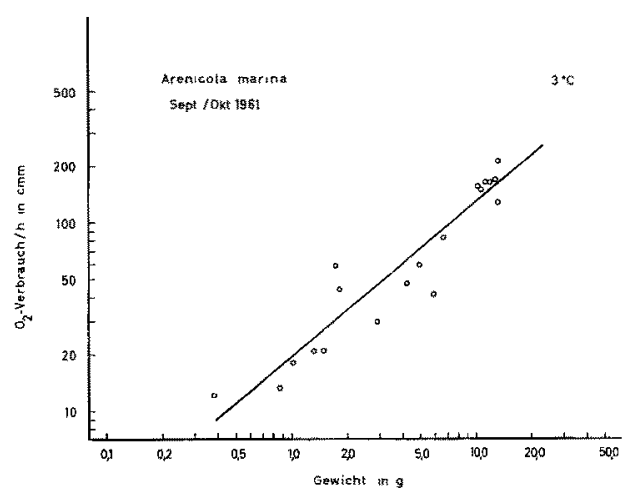

Abb. $2 b$

sagt - nur im allgemeinen und wir werden gerade bei Arenicola Abweichungen von dieser Regel kennenlernen.

Die vorliegenden Untersuchungen an Arenicola haben gezeigt, daß die Stoffumsetzungen im Organismus nicht zwangsweise den einwirkenden Temperaturen unterworfen sind, sondern daß der Organismus die Geschwindigkeit der Umsetzung in gewissen Grenzen zu steuern vermag.

Arenicola muß wohl als ein eurythermer Organismus bezeichnet werden, auch wenn er durch seine unterirdische Lebensweise vor extremsten Temperaturschwankun-

Tabelle 4

Atmungswerte von Arenicola marina. Messungen im Mai 1962, Helgoland.

Durchschnittlicher Sauerstoffyerbrauch in $\mathrm{cmm} / \mathrm{h}$

\begin{tabular}{|crrrrrrr|}
\hline $\begin{array}{c}\text { Gewicht } \\
\text { in g }\end{array}$ & $3^{0} \mathrm{C}$ & $5^{0} \mathrm{C}$ & $10^{\circ} \mathrm{C}$ & $15^{\circ} \mathrm{C}$ & $20^{\circ} \mathrm{C}$ & $25^{\circ} \mathrm{C}$ & $30^{\circ} \mathrm{C}$ \\
\hline 2,37 & 67,00 & 39,50 & 105,40 & 105,33 & 111,00 & 152,50 & - \\
2,82 & 106,17 & 119,33 & 138,83 & 192,63 & 194,75 & 412,00 & 294,17 \\
3,12 & 92,50 & 94,75 & 159,50 & 177,13 & 158,00 & 254,50 & 290,00 \\
3,21 & 131,00 & 46,75 & 118,33 & 221,84 & 107,33 & 547,33 & - \\
3,22 & - & 23,50 & 31,60 & 95,90 & - & - & - \\
3,24 & - & 35,23 & 42,20 & 107,00 & - & - & - \\
3,38 & 111,33 & 100,00 & 134,83 & 202,50 & 153,00 & - & - \\
3,60 & - & 11,75 & 30,40 & 105,40 & - & - & - \\
4,00 & - & 83,50 & 180,00 & 182,38 & 169,25 & 223,50 & 293,25 \\
4,65 & - & 121,75 & 181,25 & 198,37 & 263,33 & 361,00 & 165,50 \\
4,91 & 72,25 & 86,25 & 162,00 & 167,45 & 189,80 & 255,50 & 333,17 \\
4,93 & 106,25 & 64,33 & 198,20 & 195,00 & 274,40 & 301,25 & 412,83 \\
5,72 & - & 35,67 & 90,20 & 108,80 & - & - & - \\
5,95 & 61,00 & 96,75 & 219,57 & 233,13 & 277,00 & 324,00 & 299,50 \\
7,65 & 81,00 & 50,00 & 150,75 & 190,17 & 227,25 & 224,00 & - \\
8,26 & - & 162,50 & 228,33 & 254,35 & 284,00 & 323,25 & - \\
8,50 & - & 62,50 & 114,25 & 191,88 & - & - & - \\
10,62 & 122,75 & 158,50 & 273,67 & 416,87 & 489,00 & 649,00 & 598,33 \\
14,60 & - & 212,25 & 276,00 & 472,75 & 525,75 & 690,33 & - \\
18,76 & - & 261,33 & 398,00 & 538,34 & 489,75 & 616,00 & - \\
\hline
\end{tabular}




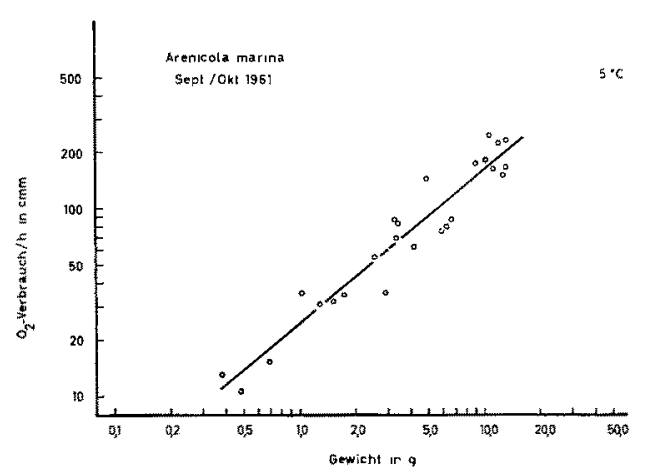

Abb. 2c

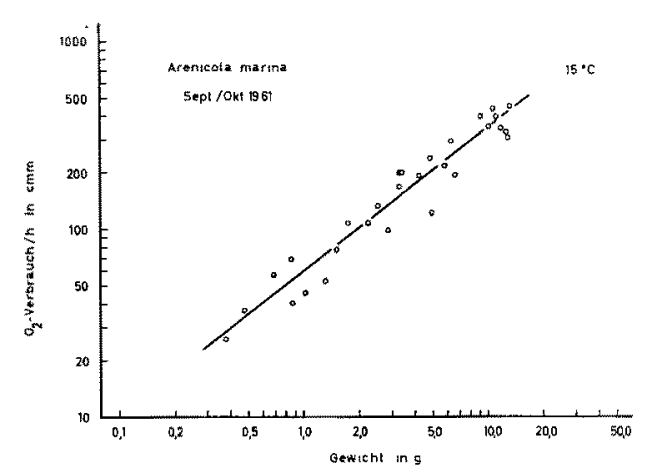

Abb. $2 \mathrm{e}$

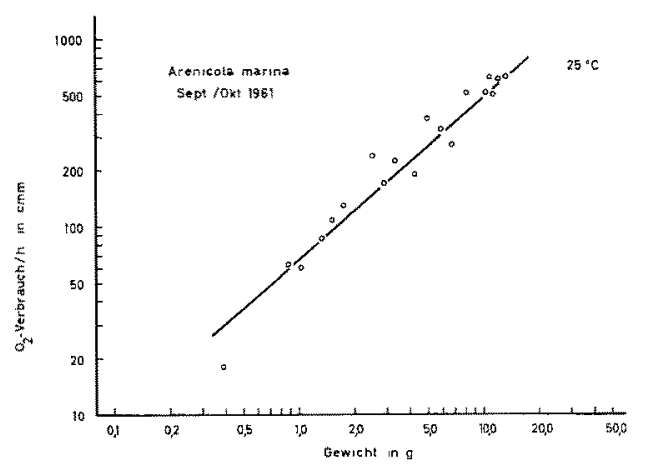

Abb. $2 \mathrm{~g}$

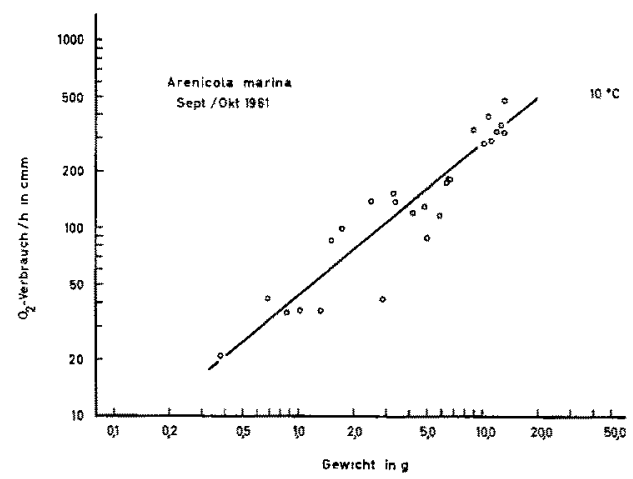

Abb. 2d

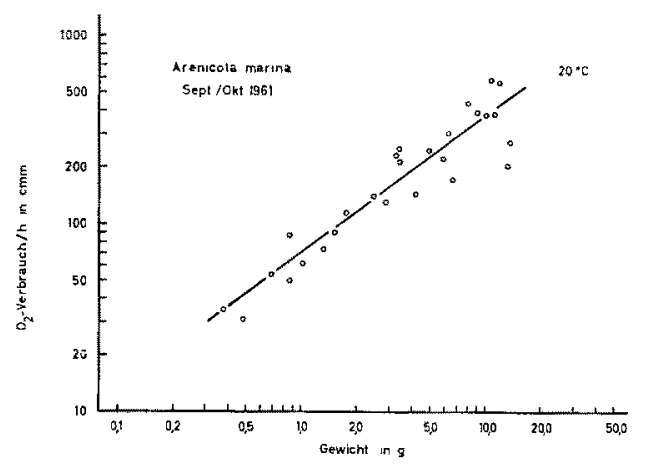

Abb. $2 f$

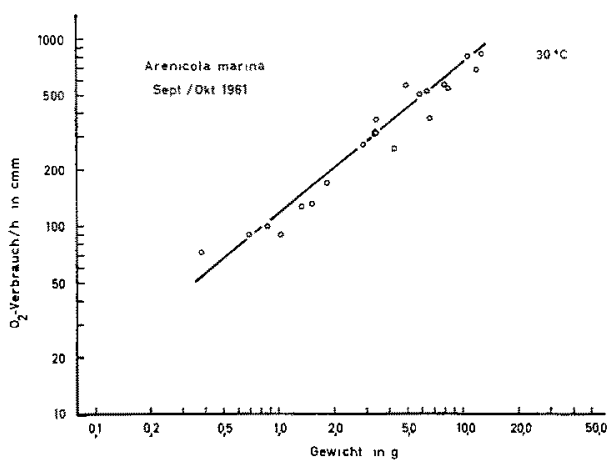

Abb. 2h

Abb. 2: Größenabhängigkeit der Atmung von Arenicola bei verschiedenen Temperaturen. Versuche vom Herbst 1961. Eingezeichnet sind die berechneten Regressions-Geraden

gen geschützt ist. Im Sommer besteht für ihn die Möglichkeit, daß das über den heißen Wattboden einströmende Wasser sich stark erwärmt hat und dadurch auch in seinem. Wohngang die Temperaturen durch das eingepumpte Wasser auf $25^{\circ} \mathrm{C}$ und mehr steigen. Umgekehrt sinkt im Winter die Temperatur wohl auch im Wattboden auf $0^{\circ} \mathrm{C}$ 
und weniger - wenigstens zeitweise - ab. Einer direkten Vereisung des Wattbodens scheint Arenicola allerdings nach den Beobachtungen von Werner (1954) auszuweichen.

Zwischen diesen Extremtemperaturen dürfte sich das Leben des Wattwurmes abspielen. Dementsprechend wurde die Temperaturabhängigkeit der Atmung von Arenicola zwischen $3^{\circ} \mathrm{C}$ (teilweise auch $2^{\circ} \mathrm{C}$ ) und $30^{\circ} \mathrm{C}$ gemessen. Leider reichte die Leistung des benutzten Kühlaggregates nicht aus, mit den Meßtemperaturen noch tiefer zu gehen. Gerade der untere Temperaturbereich ist zur Charakterisierung des Temperatureinflusses auf biologische Objekte besonders wichtig. Temperaturen von $35^{\circ} \mathrm{C}$ ertrugen die Würmer in keinem Falle. Im Frühjahr ging schon die Mehrzahl bei $30^{\circ} \mathrm{C}$ im Verlauf der Messungen ein.

Die Wirkung der Temperatur auf die Atmungsgröße von Arenicola bietet ein sehr komplexes Bild. Sie beeinflußt nämlich nicht nur die Atmungsgröße, sondern auch die Form ihrer Größenabhängigkeit. Außerdem zeigen sich auch jahreszeitliche Unterschiede. Aus diesem Grund müssen die Sommerversuche getrennt von den Versuchsreihen des Frühjahres behandelt werden. Die Meßreihen vom Herbst 1961 geben dadurch, daß hier Würmer einer Gewichtsspanne von 1:35 untersucht werden konnten, ein recht zuverlässiges Bild. In den Grundzügen finden sich entsprechende Verhältnisse, auch in den Frühjahrsversuchen und erfahren durch sie eine Bestätigung.

Betrachten wir zunächst in den Tabellen 2 und 3 die für die einzelnen Temperaturen gefundenen Durchschnittswerte, so erkennen wir, daß der Sauerstoffverbrauch der Würmer im ganzen genommen mit ansteigender Temperatur zunimmt. Die rohen Versuchswerte sind aber für eine direkte Auswertung ungeeignet, weil sich bei ihnen Temperaturwirkung und Größenfunktion überlagern. Aus diesem Grund haben wir für die einzelnen Temperaturstufen die Parameter der allometrischen Funktion berechnet. Sie sind in Tabelle 5 zusammengestellt. Um die Anwendbarkeit der allometrischen Funktion deutlicher zu machen, sind in den Abbildungen $2 \mathrm{a}$ bis $\mathrm{h}$ und $3 \mathrm{a}$ bis e die Versuchswerte graphisch in ein doppellogarithmisches Koordinatensystem eingezeichnet worden. Die Gerade in den einzelnen Abbildungen gibt die Lage der berechneten Ausgleichsgeraden wieder, die der Auswertung der Versuche zugrunde liegt. Man erkennt, daß sie sich in fast allen Fällen recht gut den Versuchswerten anschmiegt. Man ist also berechtigt, die nach der allometrischen Formel berechneten Parameter der Auswertung der Ergebnisse zugrundezulegen.

Dadurch, daß Exponent $\alpha$ für die verschiedenen Temperaturen nicht identisch ist, zeigen die Ausgleichsgeraden bei der graphischen Darstellung verschiedene Steigungen. Es ergibt sich hieraus, daß die Temperaturfunktion für kleine Würmer eine andere Gestalt hat als für große Würmer. Die Kenntnis der Parameter der allometrischen Funktion gestattet, die Temperaturfunktion für alle beliebigen Wurmgrößen darzustellen. Hier sei nur die Auswertung für Würmer von $1 \mathrm{~g}$ Gewicht und $10 \mathrm{~g}$ Gewicht durchgeführt. Die mittlere Atmungsgröße der Würmer von $1 \mathrm{~g}$ Gewicht ist in den Faktor a der allometrischen Gleichung gegeben, deren Werte man für die verschiedenen Temperaturen und Jahreszeiten in Tabelle 5 findet. Entsprechend der allometrischen Gleichung $\log \mathrm{O}_{2}$-Verbrauch $=\log b+\alpha \log$ Gewicht erhält man den Logarithmus des Sauerstoffverbrauches für $10 \mathrm{~g}$ Würmer, wenn man zum $\log \mathrm{b}$ den Wert für $\alpha$ addiert, $\mathrm{da}$ 


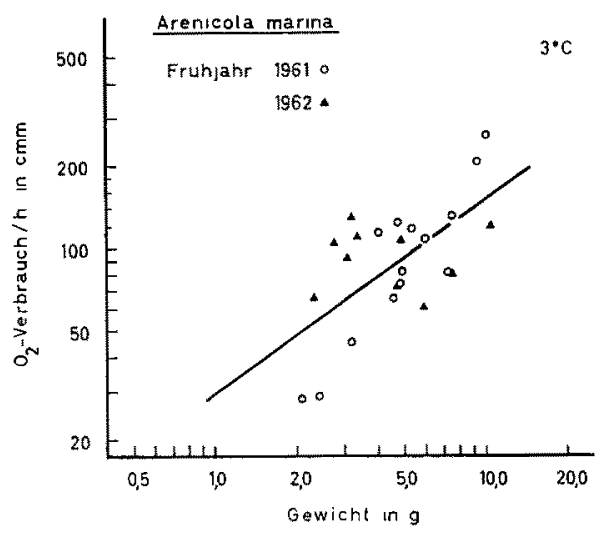

Abb. 3a

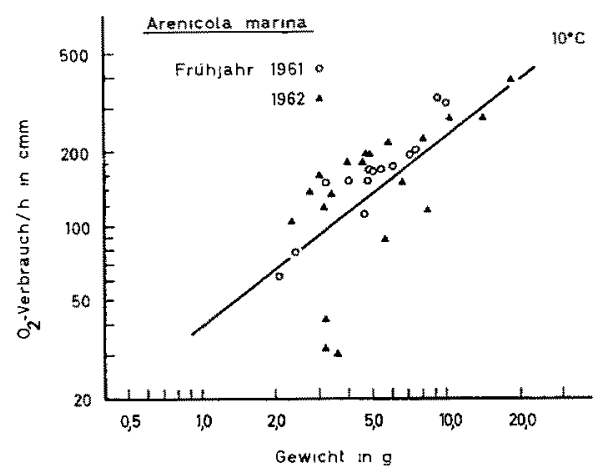

Abb. $3 c$

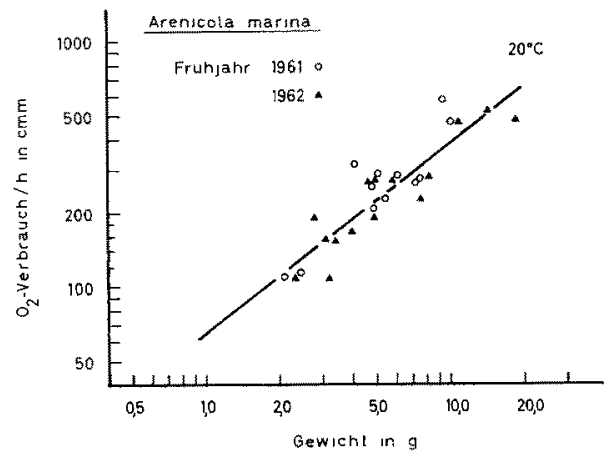

Abb. $3 e$

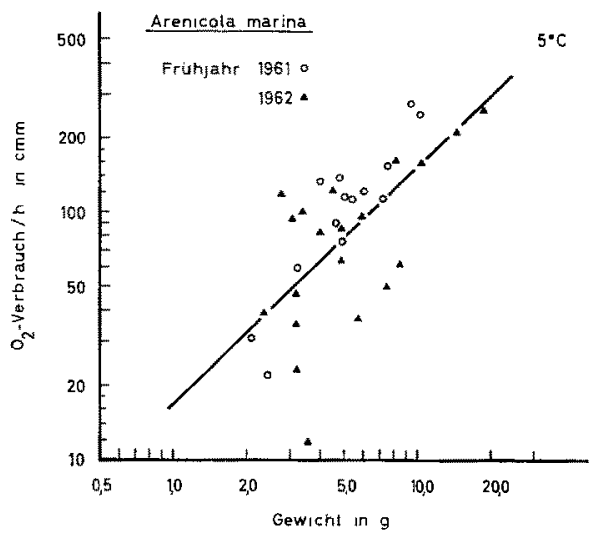

Abb. 3b

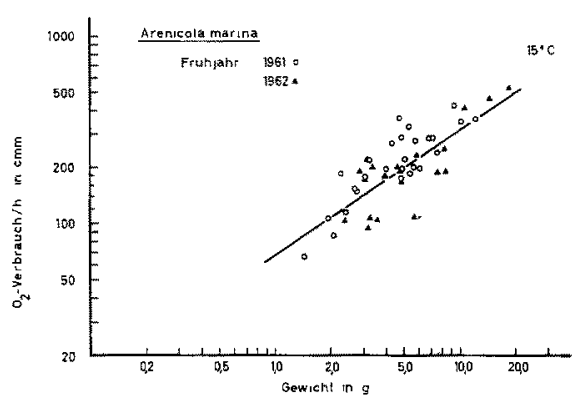

Abb. $3 d$

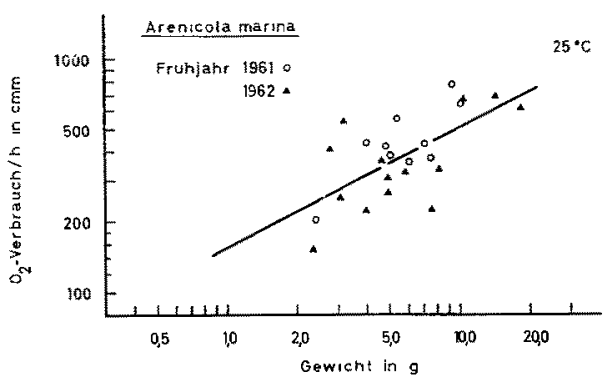

Abb. $3 f$

Abb. 3: Größenabhängigkeit der Atmung von Arenicola bei verschiedenen Temperaturen. Versuche vom April/Mai 1961, Versuche vom Mai/Juni 1962 
ja $\log 10=1$ ist. Die auf diese Weise berechneten Werte sind in Tabelle 6 zusammengestellt.

Behandeln wir zunächst die Temperaturfunktion der kleinen Würmer, deren Sauerstoffverbrauch in der Größe b direkt seinen Ausdrudk findet. Tabelle 5 zeigt uns, daß erwartungsgemäß der Wert für $b$ mit zunehmender Temperatur höher wird, aber der Anstieg erfolgt nicht gleichmäßig. Während nämlich zwischen $2^{\circ} \mathrm{C}$ und $10^{\circ} \mathrm{C}$, also bei einer Temperatursteigerung um $8^{\circ}$, der Sauerstoffverbrauch von 13,4 auf 42,3 $\mathrm{cmm} / \mathrm{h}$ zunimmt, also um etwa den Faktor 3, ist der Steigerungsfaktor zwischen $10^{\circ} \mathrm{C}$ und $25^{\circ} \mathrm{C}$, also über $15^{\circ} \mathrm{C}$, nur etwa 1,5 - von 42,3 auf $66,8 \mathrm{cmm} / \mathrm{h}$. Dieser außerordentlich niedrige Temperaturquotient kommt offensichtlich dadurch zustande, daß $z$ wischen $20^{\circ} \mathrm{C}$ und $25^{\circ} \mathrm{C}$ eine Reduktion des Sauerstoffverbrauches stattfindet. Aber selbst wenn man diese Verminderung nicht berücksichtigt, so verdoppelt sich $z$ wischen $10^{\circ} \mathrm{C}$ und $20^{\circ} \mathrm{C}$ der Sauerstoffverbrauch nicht einmal, bleibt also hinter dem Normalwert der RGT-Regel zurück. Oberhalb $25^{\circ} \mathrm{C}$ steigt der Sauerstoffverbrauch dann wieder sehr stark auf $115,8 \mathrm{cmm} / \mathrm{h}$ an.

Die Temperaturfunktion ist also gekennzeichnet durch die Unstetigkeit bei $25^{\circ} \mathrm{C}$, wo die an sich zu erwartende Stoffwechselsteigerung ausbleibt. Es resultiert hieraus in dem weiten Temperaturbereich zwischen $10^{\circ} \mathrm{C}$ und $25^{\circ} \mathrm{C}$ eine nur in engen Grenzen schwankende Atmungsgröße. Damit dürfte auch der übrige Stoffwechsel und vor allem die Aktivität des Wurmes relativ temperaturunabhängig in diesem Bereich sein. Da diese Temperaturspanne zusammenfällt mit den im Spätsommer zu erwartenden Wassertemperaturen, erscheint der hier im Laboratoriumsversuch zutage getretene Regulationsmechanismus biologisch sinnvoll.

Berechnet man den Faktor b der allometrischen Gleichung (Tab. 5), so kann seine Temperaturabhängigkeit nicht überraschen, da er ja direkt ein Ausdruck für den Sauerstoffverbrauch ist. Erstaunlicher ist die offensichtlich zutage tretende Abhängigkeit des Exponenten $\alpha$ von der Temperatur. Es steht dieser Befund allerdings nicht isoliert, so fand Jов (1955) bei Salvelinus, daß der Wert von $\alpha$ temperaturabhängig ist, und das gleiche berichtet Vernberg (1959) neuerdings für die Atmung von Uca-Arten.

Die Gewichtsspanne der Herbstversuche von 1961 war hinreichend, um mit hoher Sicherheit den Wert des Exponenten $\alpha$ (Tabelle 5) zu bestimmen. Für die Zuverlässigkeit dieser Messungen spricht auch die Konstanz seines Wertes von 0,81-0,82 für die Temperaturen $3^{\circ} \mathrm{C}, 5^{\circ} \mathrm{C}$ und $10^{\circ} \mathrm{C}$; bei $15^{\circ} \mathrm{C}$ sinkt b auf 0,75 , erreicht bei $20^{\circ} \mathrm{C}$ ein Minimum von 0,71 und steigt dann bei $25^{\circ} \mathrm{C}$ wieder auf $0,88 \mathrm{bzw} .0,77$ bei $30^{\circ} \mathrm{C}$ an. Der letzte Wert ist, ebenso wie der Wert von 0,90 bei $2^{\circ} \mathrm{C}$, angesichts der großen Schwankungsbreite der Atmungswerte vielleicht nicht ganz sicher.

Verbietet schon die Vielzahl der Messungen, die in den Zahlen verarbeitet sind, in dem Ergebnis einen Zufall zu sehen, so gewinnt es noch dadurch an Gewicht, daß wir entsprechende Erscheinungen auch bei den Frühjahrsmessungen beobachten können (Tab. 3 und 4), allerdings um $5^{\circ}$ verschoben. Der Betrachtung der Ergebnisse legen wir die Werte zugrunde, die sich aus der gemeinsamen Verarbeitung beider Versuchsreihen von 1961 und 1962 ergeben (Tab. 5). Die größere Zahl der Einzelwerte bietet dadurch einen gewissen Ausgleich für die engere Gewichtsspanne (1:8) der untersuchten Wurmgrößen. Es soll hier unberücksichtigt bleiben, daß die beiden Versuchsreihen weder zeitlich genau übereinstimmen, noch sich die Meßtemperaturen ganz deckten. 


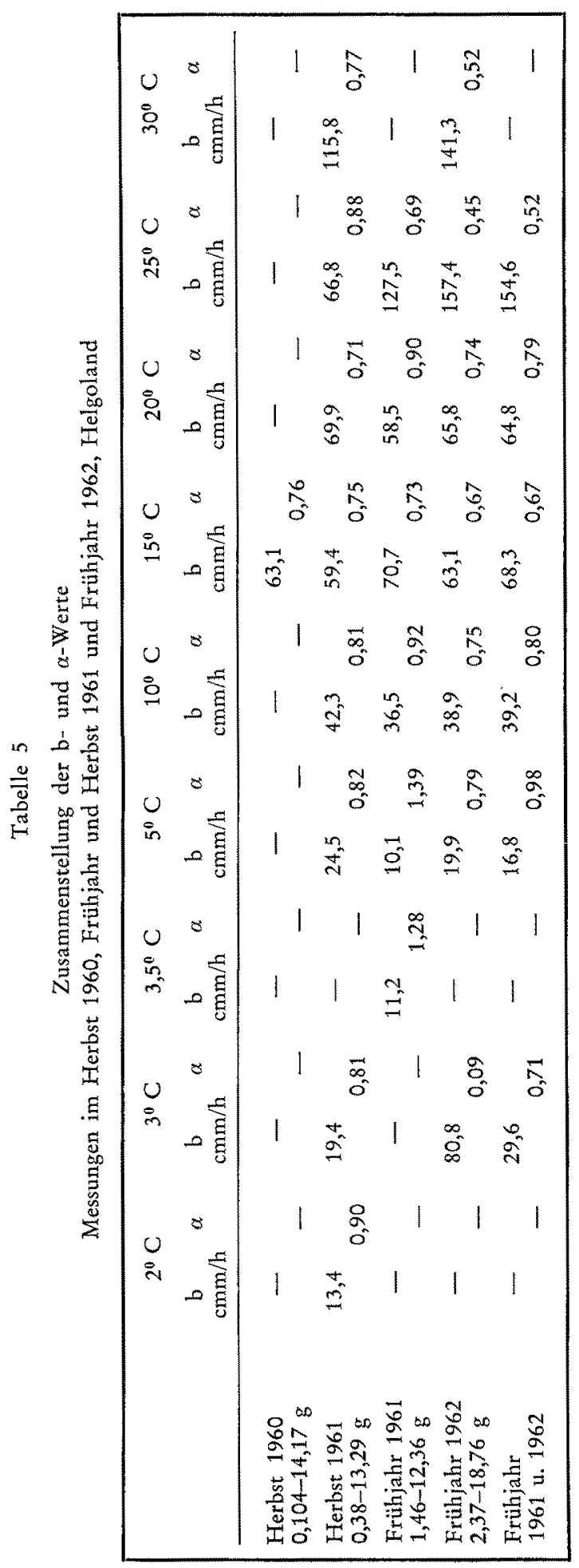


Bei den Frühjahrsversuchen trat eine zweite Unstetigkeit der Temperaturfunktion bei den tiefen Temperaturen auf, indem bei $3^{\circ} \mathrm{C}$ bzw. 3,50 $\mathrm{C}$ in zahlreichen Fällen eine Atmungssteigerung erfolgte, die sich auch in dem Durchschnittswert auswirkt. Durch diese Unstetigkeit wird der untere steile Anstieg in die Temperaturstufe zwischen $5^{\circ} \mathrm{C}$ und $10^{\circ} \mathrm{C}$ verlegt, für die sich der Sauerstoffverbrauch bei nur $5^{\circ} \mathrm{C}$ Temperatursteigerung mehr als verdoppelt. Zwischen $10^{\circ} \mathrm{C}$ und $20^{\circ} \mathrm{C}$ findet sich nur ein geringer Anstieg der Atmungsintensität - wieder durch eine Unstetigkeit -, die aber schon bei $20^{\circ} \mathrm{C}$ auftritt.

Im Durchschnitt ergibt sich bei $20^{\circ} \mathrm{C}$ eine Atmungsgröße von $64,8 \mathrm{cmm} / \mathrm{h}$ gegenüber $68,3 \mathrm{cmm} / \mathrm{h}$ bei $15^{\circ} \mathrm{C}$. Auf den Bereich mit flachem Anstieg folgt wieder eine sehr steile Atmungszunahme - noch viel ausgesprochener als bei den Sommertieren - allerdings im Frühjahr schon zwischen $20^{\circ} \mathrm{C}$ und $25^{\circ} \mathrm{C}$. Bei den wenigen Würmern, die in dieser Jahreszeit die Messungen bei $30^{\circ} \mathrm{C}$ überstanden, zeigte sich für diese letzte Stufe kein Anstieg der Atmungsgröße mehr, sondern nur noch eine leichte Verminderung, doch kann dem Wert von $141,2 \mathrm{cmm} / \mathrm{h}$ angesichts des Todes zahlreicher Würmer bei dieser Temperatur kein Gewicht mehr beigemessen werden.

Die in den Frühjahrswerten zutage getretene Verschiebung der Unstetigkeit und der Maximalatmung um $5^{\circ} \mathrm{C}$ niedrigere Werte betrifft auch die Exponenten $\alpha$. Das in den Sommerversuchen bei $20^{\circ} \mathrm{C}$ liegende Minimum von 0,71 findet sich im Frühjahr schon bei $15^{\circ} \mathrm{C}$ und erreicht hier sogar den Wert 0,67 , der eine oberflächenproportionale Atmung charakterisiert. Ober- und unterhalb von $15^{\circ} \mathrm{C}$ liegen die Exponenten wieder höher, mit Ausnahme des Exponenten bei $25^{\circ} \mathrm{C}$, der sogar extrem niedrig liegt. Die Temperaturbeziehung der Atmungswerte verschiebt sich jahreszeitlich also im Sinne der normalen Umgebungstemperaturen.

Im Endergebnis resultiert aus den beobachteten Unstetigkeiten nicht nur eine von der Temperatur wenig abhängige, sondern auch eine im Verlauf des ganzen Jahres ziemlich konstante Atmungsgröße zwischen $10^{\circ} \mathrm{C}$ und $20^{\circ} \mathrm{C}$; eine Tatsache, die ökologisch offensichtlich sehr bedeutungsvoll für den Wurm ist.

Auf den Temperaturbereich mit gedrosselter Stoffwechselintensität folgt bei den Sommertieren über $25^{\circ} \mathrm{C}$, ebenso wie bei den Frühjahrstieren über $20^{\circ} \mathrm{C}$, ein besonderer Anstieg der Atmungsgröße, der anscheinend im Frühjahr noch steiler ist als im Sommer. Der steile Anstieg kennzeichnet die obere Grenztemperatur, die die Würmer ohne offensichtliche Schädigung ertragen kömnen. Steigert man die Temperatur um weitere $5^{\circ} \mathrm{C}$, so zeigen sie fast ausnahmslos Anzeichen ernstlicher Schädigung, wie Austritt von Blut, Turgorverlust und morphologische Deformationen oder sie überleben das Ende der Versuche nicht mehr. Beachtenswerterweise lag die letale Temperatur im Frühjahr $5^{\circ} \mathrm{C}$ tiefer als im Herbst. Auch hier erkennt man wieder die Einpassung der physiologischen Reaktionen in die natürlicherweise gegebenen Temperaturbedingungen.

Diese Ausführungen beziehen sich auf die durch die Größe b der allometrischen Gleichung ausgedrückte Atmung eines Wurmes von $1 \mathrm{~g}$ Gewicht. Es lassen sich diese Befunde nicht ohne weiteres auf größere Würmer anwenden, da durch die Inkonstanz des Wertes von $\alpha$ die Atmungsgröße bei verschiedenen Temperaturen nicht parallel zum Gewicht zunimmt. Die Temperaturfunktion hat bei Würmern von abweichendem Gewicht eine andere Gestalt. Um diese Tatsache vor Augen zu führen und die daraus sich ergebenden Konsequenzen zu besprechen, habe ich in Tabelle 6 die Werte zusammengestellt, die sich nach der allometrischen Formel für Würmer von $10 \mathrm{~g}$ Gewicht 
Tabelle 6

Sauerstoffverbrauch von Würmern von $10 \mathrm{~g}$ Gewicht in $\mathrm{cmm} / \mathrm{h}$ berechnet nach der Formel: $\log \mathrm{O}_{2}$-Verbr. $=\log \mathrm{b}+\alpha$

\begin{tabular}{|lcccccccc|}
\hline Temperatur & $2^{0} \mathrm{C}$ & $3^{0} \mathrm{C}$ & $5^{\circ} \mathrm{C}$ & $10^{\circ} \mathrm{C}$ & $15^{\circ} \mathrm{C}$ & $20^{\circ} \mathrm{C}$ & $25^{\circ} \mathrm{C}$ & $30^{\circ} \mathrm{C}$ \\
\hline Herbst & 106 & 125 & 162 & 273 & 334 & 359 & 507 & 683 \\
Frühjahr & & 152 & 160 & 247 & 320 & 400 & 516 & \\
\hline
\end{tabular}

ergeben. Wie man aus der Zusammenstellung ersieht, zeigen sich in dem sehr weiten Temperaturbereich zwischen $3^{\circ} \mathrm{C}$ und $25^{\circ} \mathrm{C}$ nur recht geringe Unterschiede für die mittleren Atmungswerte der größeren Würmer im Frühjahr und im Herbst. Erheblich höher liegen bei den größeren Frühjahrstieren die Atmungswerte bei $3^{\circ} \mathrm{C}$ und bei $20^{\circ} \mathrm{C}$. Für die tiefere Temperatur, die in dieser Jahreszeit noch in der Nähe der normalen Milieutemperatur liegt, darf man wohl annehmen, daß hier ein zu starkes $\mathrm{Ab}$ sinken der Aktivität verhindert werden soll. Für den höheren Atmungswert bei $20^{\circ} \mathrm{C}$ ist zu vermuten, daß die im Herbst bei dieser Temperatur wirksame Regulation fehlt, weil im. Frühjahr so hohe Wassertemperaturen nicht zu erwarten sind. Bei den kleineren Würmern zeigr sich allerdings auch bei dieser Temperatur eine sehr ausgesprochene Regulation.

Im ganzen genommen muß man feststellen, daß die Atmungsgröße von Arenicola keine eindeutigen Anderungen im Verlaufe des Jahres erfährt. Die jahreszeitlichen Anpassungen beschränken sich auf eine Verschiebung der Temperaturbereiche, in denen Regulationen des Stoffwechsels erfolgen.

\section{DISKUSSION}

Die Grundlage der vorliegenden Messungen bildete die Bestimmung der Größen $\alpha$ und $\mathrm{b}$ der allometrischen Gleichung $\mathrm{y}=\mathrm{b} \cdot \mathrm{w}^{\alpha}$ zur eindeutigen Kennzeichnung der Atmungsgröße von Arenicola unter der Einwirkung verschiedener Temperaturen und zu zwei verschiedenen Jahreszeiten. Bei der mathematischen Verarbeitung biologischer Meßdaten erhebt sich der Einwand, wie weit ein solches Vorgehen berechtigt ist. Die Voraussetzung einer sinnvollen mathematischen Behandlung ist die Reproduzierbarkeit der Meßergebnisse, die die Atmungsmessungen an Arenicola demonstrieren konnten. Das gilt einmal für die Ergebnisse verschiedener Autoren, die wenigstens größenordnungsmäßig übereinstimmen (KRÜGER 1958), in verstärktem Maße gilt es aber für Messungen unter identischen Verhältnissen, wie der Vergleich der Versuche vom Herbst 1960 und 1961 in dieser Arbeit zeigte. Die nur geringfügigen Differenzen der Parameter der allometrischen Gleichung, die wir hierbei fanden, zeigen die grundsätzliche Ubereinstimmung der Ergebnisse.

Der Exponent $\alpha$, der die Gestalt der Größenabhängigkeit des Stoffwechsels charakterisiert, wurde vielfach als eine Größe angesehen, die für größere und kleinere systematische oder biologische Gruppen gültig ist. VON BERTALANFFY vor allem brachte den Exponenten $a$ in Beziehung zu systematischen Kategorien, während LuD- 
wIG (1956) Zusammenhänge mit dem Typus der Atmungsorgane suchte. Für beide Anschauungen bietet sich angesichts der starken Variabilität des Exponenten keine Stütze. Leider scheint Arenicola der erste polychaete Annelide zu sein, für den die Größenbeziehung des Stoffwechsels quantitativ untersucht wurde, so daß aus dem Bereich der engeren Verwandtschaft keine Vergleichswerte zur Verfügung stehen.

Für die oligochaeten Anneliden liegt eine Reihe von Angaben vor. Unter ihnen steht der Befund von Müller (1943) isoliert, die aus ihren Messungen beim Regenwurm auf eine gewichtsproportionale Atmung schloß $(\alpha=1)$. Alle anderen Autoren beschreiben oberflächenproportionale Atmung, und zwar MENDES \& VALENTE (1953) bei tropischen Regenwürmern, KRÜGER (1955) bei Eisenia foetida, SAROJA (1959) bei indischen Regenwürmern und schließlich fand SCHWEER (1959) das gleiche bei Hirudineen, die ja den Oligochaeten sehr nahestehen.

Die in Abhängigkeit von der Temperatur nicht unerheblich schwankenden Werte für $\alpha$ machen es für Arenicola unmöglich, einen bestimmten Wert herauszustellen (Tab. 5). Die unteren Werte liegen in der Nähe von 0,67 , wobei ich die zum Teil noch kleineren Werte bei Temperaturen von $25^{\circ} \mathrm{C}$ und mehr außer Betracht lassen möchte. Diese niederen Werte kennzeichnen also eine oberflächenproportionale Atmung, die bei bestimmten Temperaturen auftritt. Im allgemeinen aber liegen die Exponenten höher, obne jedoch den Wert 1 für eine gewichtsproportionale Atmung zu erreichen. Arenicola gehört also zu der Gruppe von Organismen, deren Atmungsgröße zwischen einer Oberflächen- und Gewichtsproportionalität liegt, gekennzeichnet durch Exponenten $z$ wischen 0,66 und 1,00. In neuerer Zeit werden immer mehr Beispiele bekannt, die diesen Zwischentypus repräsentieren, ohne daß sich bestimmte Beziehungen zu systematischen Kategorien oder biologischen oder physiologischen Gegebenheiten erkennen lassen. Die bei Arenicola zutage getretene Variabilität des Exponenten, wie sie auth schon von anderen Objekten bekannt wurde, entkleidet ihn überdies in jeder Beziehung des Charakters einer grundlegenden Größe.

Die auch bei Evertebraten sehr verbreitet auftretende Stoffwechselreduktion im Verlaufe des Wachstums, die in vielen Fällen sogar oberflächenproportional ist, hat man kausal zu deuten versucht. Nachdem durch das Vorkommen der Stoffwechselreduktion bei poikilothermen Tieren die Rusnersche Theorie mit ihrer Verknüpfung von Stoffwechsel und Wärmeabgabe an der Oberfläche widerlegt war, hat man den beobachteten mathematischen Zusammenhang auf andere Flächen zu beziehen versucht. Man wies darauf hin, daß es Oberflächen sind, die dem Stoffaustausch zwischen dem Organismus und seiner Umwelt dienen und daß hieraus die quantitative Beziehung $z$ wischen Stoff wechsel und Oberflächenentwicklung resultiert.

So anschaulich diese Deutung auch auf den ersten Blick erscheint, so hatte ich mich schon 1940 gegen sie gewandt. Die Versuche an Arenicola konnten mich in meiner Auffassung nur bestärken, denn es ist wohl kaum anzunehmen, daß der offensichtlich mit der Temperatur variierenden Größenfunktion entsprechende Änderungen der Oberfläche des Wurmes parallel laufen.

Wenn man überdies den einfachen Zusammenhang zwischen Stoffwechselgrößen und Oberflächenentwicklung herausstellt, so würde das bedeuten, daß die Oberfläche als limitierender Faktor für den Stoffwechsel auftritt. Diese Annahme ist unwahrscheinlich, da alle unsere Stoffwechselmessungen - nach Möglichkeit - an ruhenden 
Tieren vorgenommen werden. Aktivität der Tiere steigert den Stoffwechsel erheblich, also müssen schon aus physiologischen Gründen die Oberflächen eines Organismus stärkeren Belastungen gewachsen sein, können daher auch nicht als limitierender Faktor auftreten.

Untersucht man übrigens die Atmung isolierter Hautstücke von Arenicola - Versuche, über die an anderer Stelle berichtet werden soll -, so zeigen auch diese Hautstücke die bei den intakten Würmern gefundene Stoffwechselreduktion im Verlauf des Wachstums, obwohl nicht anzunehmen ist, daß in diesem Falle die Sauerstoffversorgung durch die Oberflächenentwicklung limitiert ist.

Der Exponent $\alpha$ drückt nach meiner Auffassung nur eine während des Wachstums eintretende gesetzmäßige Verminderung der Leistungsfähigkeit des Atmungssystems aus. Die Anderungen von $\alpha$ bei verschiedenen Temperaturen sind nach dieser Auffassung ein Ausdruck für den Einfluß der Temperatur auf das Atmungssystem, der nicht für alle Tiergrößen die gleiche Form hat. Besonders beachtenswert sind die Minima des Exponenten $\alpha$ bei $15^{\circ} \mathrm{bzw} .20^{\circ} \mathrm{C}$, denn der Exponent $\alpha$ stellt das Verhältnis des Sauerstoffverbrauchs zum Gewicht dar. Je kleiner $\alpha$ ist, um so geringer ist der Sauerstoffverbrauch je Gewichtseinheit. Die Minima sind also der Ausdruck für einen optimalen Wirkungsgrad der Atmung bei diesen Temperaturen.

Die Wirkung der Temperatur auf den Sauerstoffverbrauch von Arenicola ist gekennzeichnet durch eine Reihe von Unstetigkeiten, deren Effekt es ist, die Atmung unter verschiedensten Bedingungen nahezu konstant zu halten. Da die Aktivität des Wurmes ohne Zweifel in engem Zusammenhang zur Atmungsgröße steht, bedeutet es zugleich, daß sie während des ganzen Jahres unter den in seinem Biotop herrschenden Temperaturbedingungen nahezu konstant ist. Es kann keinem Zweifel unterliegen, daß dieser Umstand biologisch für den Wurm äußerst bedeutungsvoll ist.

Dagegen bereiten die Unstetigkeiten in der Temperaturfunktion der Atmung sowohl dem Verständnis als auch einer mathematischen Bearbeitung große Schwierigkeiten. Auf eine Bestimmung von $\mathrm{Q}_{10^{-}}$Werten habe ich bewußt verzichtet, da sie in keiner Weise konstant wären und nicht einmal einen gleichmäßigen Gang zeigen würden. Das gleiche Bedenken besteht gegen eine Anwendung der Arrhenuus-Funktion.

Im vergangenen Jahr (1961) hatte ich zur Darstellung biologischer Temperaturfunktionen angesichts der Unzulänglichkeit der bisher üblichen Darstellungsweise eine neue Funktion vorgeschlagen und ihre Anwendbarkeit und Eignung an einer Reihe von Beispielen demonstrieren können. Grundsätzlich handelt es sich bei dem neuen Ausdruck:

$$
y_{t}=\cdots \cdots \frac{m}{n^{t-z}}
$$

um die Arrhentus-Formel, die nur etwas vereinfacht ist und in der an die Stelle der absoluten Nulltemperatur eine höhere Temperatur $z$ tritt. Der Verlauf der 'Temperaturfunktion wird in Gleichung (2) durch die Parameter $n$ und $z$ wiedergegeben, wobei n eine sehr große Zahl ist. $\mathrm{m}$ stellt mathematisch die maximale Atmungsgröße dar. $y_{t}$ ist der Sauerstoffverbrauch bei der Meßtemperatur $t$.

Die Prüfung des Zutreffens von Gleichung (2) auf eine Serie von Versuchswerten 
erfolgt am einfachsten in der Weise, daß man in einem semilogarithmischen Koordinatensystem die Logarithmen der Meßwerte den auf der Abszisse eingetragenen Werten von $1 / t-z$ zuordnet. Man varilert $z$ so lange, bis alle Meßpunkte angenähert auf einer Geraden liegen. Wenn die Größe der Nullpunkttemperatur auf diese Art bestimmt ist, lassen sich die anderen Parameter sehr leicht berechnen, denn es ist:

und

$$
\log n=\cdots \frac{\log y_{2}}{\frac{1}{t_{1}-z}}-\frac{\log y_{1}}{\frac{1}{t_{2}-z}} \ldots
$$

$$
\log m=\log y_{t}+\frac{1}{t-z} \log n
$$

Es wurden aber nicht die Meßwerte der einzelnen Würmer der Analyse nach Gleichung (2) unterworfen, sondern die aus der allometrischen Gleichung sich ergebenden Werte für Würmer von $1 \mathrm{~g}$ und $10 \mathrm{~g}$ Gewicht. Für die Versuche vom Herbst 1961 ließen sich die Werte für $b$ einigermaßen befriedigend darstellen, wenn man für $z$ den Wert $-20^{\circ} \mathrm{C}$ annimmt und den aus dem Rahmen der übrigen Werte vollkommen herausfallenden - absinkenden - Wert für $25^{\circ} \mathrm{C}$ nicht berücksichtigt. Dieser $z$-Wert würde also für Würmer von $1 \mathrm{~g}$ Körpergewicht gelten. Es interessierte mich in diesem Zusammenhang die Frage, ob die Temperaturfunktion größenabhängig ist, wie es die Versuche von Јов (1955) für Salvelinus fontinalis ergaben und für die eine genaue mathematische Analyse nach Gleichung (2) möglich war (KRüGER 1961). Ich entnahm daher aus den Kurven die Atmungswerte für Würmer von $10 \mathrm{~g}$ Gewicht und habe versucht, sie in entsprechender Weise nach Gleichung (2) darzustellen. Hierbei ergaben sich aber noch größere Schwierigkeiten als bei den Würmern von $1 \mathrm{~g}$ Gewicht, so daß für die großen Würmer noch weniger als für die kleinen ein gesicherter $z$-Wert bestimmt werden konnte. Aus diesem Grunde habe ich auch darauf verzichtet, die Parameter der Gleichung (2) elektronisch berechnen zu lassen.

Orientierungshalber sind in Abbildung 4 die Werte für die 10-g-Tiere als Funktion von $1 / t+20$ eingetragen. Wie man sieht, weichen im Temperaturbereich zwischen $15^{\circ} \mathrm{C}$ und $25^{\circ} \mathrm{C}$ die Versuchswerte unzulässig stark von den erwarteten Werten ab. Wählt man $z$ so, daß diese Werte besser erfaßt werden, so fallen die anderen Werte heraus. Offensichtlich erfolgt also bei Arenicola im Verlaufe des Wachstums eine Anderung der Temperaturfunktion, aber es ist schwierig, sie exakt zu erfassen.

Die Ursache für das Versagen der mathematischen Analyse liegt ohne $Z_{w}$ eifel in den erheblichen Unstetigkeiten in der Temperaturabhängigkeit der Atmung von Arenicola. Gleichung (2) setzt einen stetigen Zuwachs der Werte als Funktion der Temperatur voraus. Treten Unstetigkeiten auf, so kann man nur versuchen, aus dem Rahmen fallende Werte nicht $\mathrm{zu}$ berücksichtigen und möglichst zahlreiche Werte zu erfassen, bringt dadurch aber ein gewisses Maß Willkür in die mathematische Analyse.

Man könnte unter diesen Umständen grundsätzliche $Z$ weifel an dem Wert einer mathematischen Analyse biologischer Messungen hegen, doch bin ich der Uberzeugung, daß wir dazu nicht berechtigt sind. Die mathematische Auswertung hat primär die Aufgabe, biologische Funktionen möglichst eindeutig durch die Angabe der Parameter einer mathematischen Formel zu beschreiben. Ist dieses möglich, so bedeutet der mathematische Ausdruck einen ganz wesentlichen Gewinn, denn wir kennzeichnen durch ihn 
einen Kurvenverlauf mit wenigen Zahlen, an Stelle einer unübersichtlichen Wiedergabe von Zahlen und einer langatmigen Beschreibung. Solche Zahlen bilden überdies die einzig zuverlässigen Unterlagen für den Vergleich von Kurvenverläufen, der beschreibend kaum wiederzugeben ist. In Formel (2) ist $\mathrm{z}$ der Ausdruck für die Krümmung der Kurve und sein Wert kennzeichnet an sich schon charakteristische Züge des Kurvenverlaufs.

Wenn auf der einen Seite sich schon eine Reihe von biologischen Temperaturfunktionen mit Gleichung (2) darstellen ließen (KRÜGER 1961), wirkt es zunächst befremdend, daß die Versuchswerte von Arenicola offensichtlich aus dem Rahmen dieser Funktion herausfallen. Die Ursache für diese Sonderstellung von Arenicola ergab sich aus der Unstetigkeit in der Temperaturfunktion und zwar gerade in dem Temperaturbereich, der ohne Zweifel für den Wurm biologisch bedeutungsvoll ist.

Man kann diese Sonderstellung von Arenicola vielleicht mit seiner sessilen Lebensweise erklären. Wenn ein Fisch oder ein anderer frei beweglicher Organismus auf

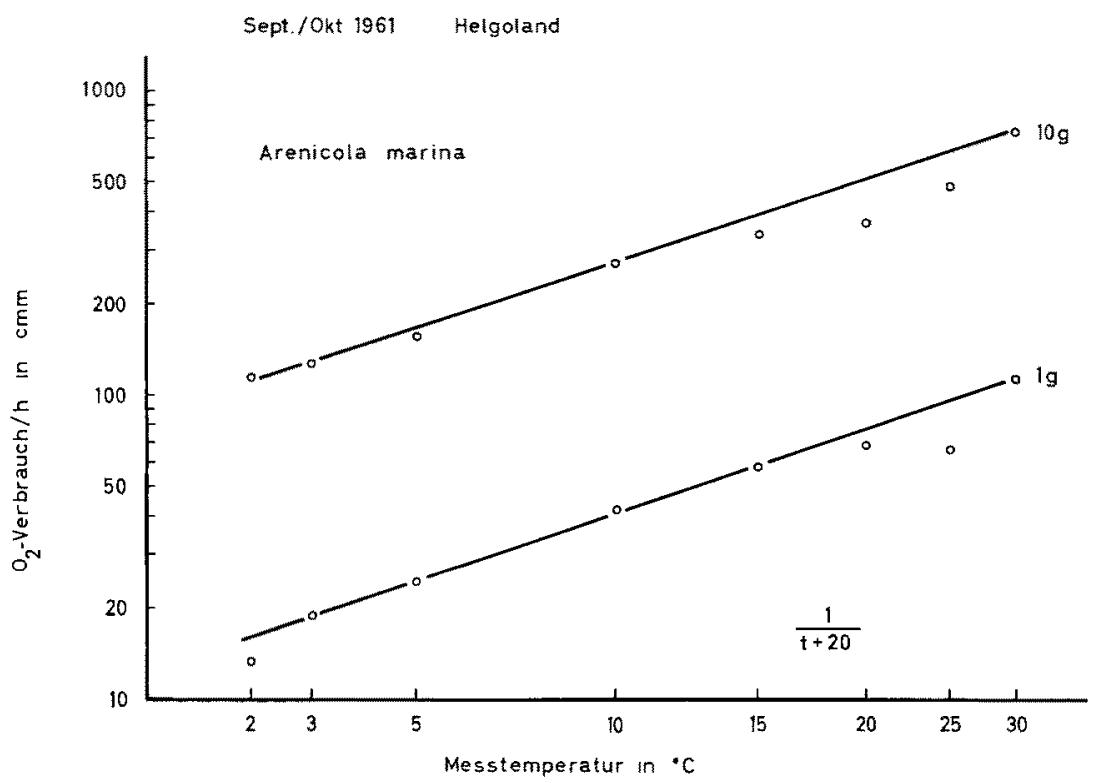

Abb. 4: Temperaturabhängigkeit des Sauerstoffyerbrauchs von Arenicola in den Herbstversuchen, dargestellt als Funktion von $\frac{1}{t+20}$. Die eingetragenen Punkte sind die Werte, die sich aus der allometrischen Formel für Individuen von $1 \mathrm{~g}$ bzw. $10 \mathrm{~g}$ Gewicht ergeben

ungünstige Temperaturbedingungen stößt, so kann er ihnen durch seine Freizügigkeit ausweichen. Ein sessiles Tier - wie Arenicola - kann das nicht und muß trotzdem seine Lebensfunktionen in vollem Umfang aufrechterhalten. Das ist nur möglich, wenn es den Ablauf der Lebensprozesse über einen weiten Temperaturbereich unabhängig von der Außentemperatur nahezu konstant halten kann. Das ist offensichtlich bei Arenicola der Fall und gerade die neuartige Darstellung der Temperaturfunktion in Abbildung 4 bringt diese Tatsache besonders deutlich zum Ausdruck. 
Unstetigkeiten in der Temperaturfunktion sind ein Kennzeichen biologischer Prozesse. Sie finden sich nicht im Bereich unbelebter Systeme, bei denen eine Temperaturerhöhung stets eine Beschleunigung temperaturabhängiger Vorgänge zur Folge hat. Im biologischen Bereich ist die beschleunigende Wirkung der Temperaturerhöhung nur eine Regel, die nicht unbedingte Gültigkeit hat.

Es liegen in der Literatur zahlreiche Hinweise auf Beobachtungen vor, in denen Organismen paradox auf Temperaturänderungen reagieren. Trotzdem hat dieses Phänomen noch nicht allgemeine Anerkennung gefunden, wie z. B. das Bedenken beweist, das WIESER (1960 p. 356) gegenüber solchen Angaben äußert. In meinem eigenen Erfahrungskreis war ich erstmals beim Schweinespulwurm (1940) auf eine solche Zone bei etwa $39^{\circ} \mathrm{C}$ aufmerksam geworden, in der eine Temperaturerhöhung keine Steigerung des Stoffwechsels zur Folge hat, während kurz oberhalb dieser Temperatur der Sauerstoffverbrauch der Würmer wieder sehr stark ansteigt. SCHMIDT (1956) hatte ähnliche "Regulationsbereiche" auch bei Insekten nachweisen können.

Bei Arenicola waren solche Unstetigkeiten in den Versuchen immer wieder in den gleichen Temperaturbereichen mit solcher Regelmäßigkeit aufgetreten, daß man an ihrer Realität nicht zweifeln kann. Leider bereitet es uns aber Schwierigkeiten, diese Unstetigkeiten $z u$ deuten, bei denen also trotz Temperaturänderung die Stoffwechselgröße sich kaum oder sogar entgegen der Erwartung ändert. Wir kennen im Bereich unserer Erfahrung im Unbelebten keinen Prozeß, der durch eine Temperaturerhöhung verzögert wird. Das gilt sowohl für chemische wie auch physikalische Prozesse.

In manchen Fällen dürfte die Konstanz der Stoffwechselwerte bei sich ändernder Temperatur auf einer Änderung der Aktivität beruhen, wenn das Tier in einer Behaglichkeitstemperatur zur Ruhe kommt. Jede Temperaturänderung nach oben oder unten kann in einem solchen Falle zu einer Stoffwechselsteigerung führen (SchmeINGEngBerding 1953, Sснмidt 1956). Ich glaube aber nicht, daß diese Art der Deutung für Arenicola zutreffend ist. Es liegt keinerlei Hinweis dafür vor, daß in dem Temperaturbereich von $20^{\circ} \mathrm{C}-25^{\circ} \mathrm{C}$ ein Aktivitätsminimum für Arenicola vorliegt, immerhin bleibt diese Frage noch experimentell zu prüfen.

\section{ZUSAMMENFASSUNG}

1. Es wird die Größenabhängigkeit des Sauerstoffverbrauchs von Arenicola bei Temperaturen $z w i s c h e n 2^{\circ} \mathrm{C}$ und $30^{\circ} \mathrm{C}$ in Versuchsserien im Frühjahr und Herbst gemessen.

2. Da der Sauerstoffverbrauch je Gewichtseinheit bei den Würmern keine konstante Größe darstellt, werden die Parameter $\alpha$ und $b$ der allometrischen Gleichung: $y=$ $b \cdot w^{\alpha}$ zur Auswertung der Versuchsergebnisse herangezogen.

3. Der Exponent $a$ hat keine konstante Größe, sondern ändert sich mit der Temperatur. Sein Wert liegt für Arenicola im allgemeinen $z$ wischen 0,7 und 0,8, was einer Zwischenstellung $z$ wischen oberflächen- und gewichtsproportionaler Atmung entspricht.

4. Der Faktor b stellt die aus der Gesamtheit dex Meßwerte - unter Berücksichtigung der Größenfunktion - berechnete Atmungsgröße eines Tieres von der Gewichts- 
einheit dar. $b$ hat daher in der quantitativen Versuchsauswertung an die Stelle der einfachen Angabe $\mathrm{O}_{2}$-Verbrauch : Gewicht zu treten.

5. Die Temperaturabhängigkeit des Sauerstoffverbrauchs von Arenicola ist durch Unstetigkeiten charakterisiert, worunter Temperaturbereiche verstanden werden sollen, in denen der Sauerstoffverbrauch erheblich über oder unter den nach der RGT-Regel zu erwartenden Werten liegt.

6. Aus diesen Unstetigkeiten erklärt sich die geringe Temperaturabhängigkeit des Stoffwechsels von Arenicola in dem Bereich zwischen $10^{\circ} \mathrm{C}$ und $20^{\circ} \mathrm{C}$. Eine zweite Unstetigkeit verhindert bei den Würmern im Frühjahr ein zu starkes Absinken des Stoffwechsels unterhalb von $5^{\circ} \mathrm{C}$. In vielen Versuchen findet man hier bei abnehmender Temperatur einen erhöhten Sauerstoffverbrauch.

7. Die Lage und Ausdehnung der Unstetigkeiten verschiebt sich im Verlauf des Jahres im Sinn der Milieu-Temperaturen.

Für die sorgfältige Durchführung der zahlreichen Atmungsmessungen und die verständnisvolle Mitarbeit bei den Auswertungen der Versuche und der Abfassung des Manuskriptes. bin ich meiner technischen Assistentin Fräulein Margret MüHL_ENkamp zu herzlichstem Dank verpflichtet.

\section{ZITIERTE LITERATUR}

Bertalanffy, v. L., 1957. Quantitative laws in metabolism and growth. Quart. Rev. Biol. 32, 217-231.

Brown, F. A., Bennett, M. F. \& Webb, H. M., 1954. Persistent daily and tidal rythms of $\mathrm{O}_{2}$-consumption in fiddler crabs. J. cell. comp. Physiol. 44, 477-506.

Hoesslin, v. H., 1888. Uber die Ursachen der scheinbaren Abhängigkeit des Umsatzes von der Größe der Körperoberfläche. Arch. Anat. Physiol., Abt. Physiol. 1888, 323-379.

Јов, S. V., 1955. The oxygen consumption of Salvelinus fontinalis. Publ. Ont. Fish. Res. Lab. 73, 1-39.

KrüGER, Fr., 1940. Die Beziehung des Sauerstoffverbrauchs zur Körperoberfläche beim Schweinespulwurm (Ascaris lumbricoides). Z. wiss. Zool. 152, 547-570.

- 1952. Über die Beziehung des Sauerstoffverbrauchs zum Gewicht bei Eisenia foetida (SAv.). Z. vergl. Physiol. 34, 1-5.

- 1955. Beiträge zur Physiologie des Hämoglobins wirbelloser Tiere. III. Zur vergleichenden Atmungsphysiologie der Enchyträiden. Z. vergl. Physiol. 37, 118-127.

- 1957. Temperatur-Regelung durch gesteuerte Kühlung. Helgol. Wiss. Meeresunters. 6, 71-75.

- 1958. Zur Atmungsphysiologie von Arnicola marina L. Helgol. Wiss. Meeresunters. 6, 193-201.

- 1960. Zur Frage der Größenabhängigkeit des Sauerstoffverbrauchs von Mytilus edulis. Helgol. Wiss. Meeresunters. 7, 125-148.

- 1961. Über den Exponenten der Temperaturfunktion biologischer Vorgänge und deren Größenabhängigkeit. Biol. Zbl. 80, 721-750.

Ludwig, W., 1956. Betrachtungen über den Energiekonsum von Tieren mit Atmungstypen von zweierlei Typ. Z. vergl. Physiol. 39, 84-88.

Mendes, E. G. \& Valente, D., 1953. The respiration of tropical earthworms. Bolm Fac. Filos. Ciênc. Univ. S Paulo zool. 18, 91-102.

MülLER, J., 1943. Weiteres zur Frage der Abhängigkeit der Atmung von der Körpergröße. Biol. Zbl. 63, 446-453.

NewelL, G. E., 1948. A contribution to our knowledge of the life history of Arenicola marina. J. Mar. biol. Ass. U. K. 27, 554-580.

- 1949. The later larval life of Arenicola marina. J. Mar. biol. Ass. U. K. 28, 635-639. 
Rameaux \& Sarrus, 1837/39. Bull. Acad. Méd. 2 u. 3 (zitiert nach v. Hoesslin).

SAROJA, K., 1959. Studies on oxygen consumption in tropical poikilotherms. II. Oxygen consumption in relation to body size and temperature in the earthworm, Megascolex mauritii, when kept submerged under water. Proc. Indian Acad. Sci. (B) 49, 183-193.

SCHMEING-ENGBERDING, F., 1953. Vorzugstemperaturen einiger Knochenfische und ihre physiologische Bedeutung. Z. Fisch. 1953, 125-155.

SchMid, G., 1956. Der Stoffwechsel der Caraben (Ins. Coleopt.) und seine Beziehung zum Wasserhaushalt. Zool. Jb. allgem. Zool. 66, 273-294.

SCHWEER, M., 1959. Untersuchungen zur vergleichenden Stoff wechselphysiologie einheimischer Sïßwasseregel (Hirudinea). Z. vergl. Physiol. 42, 20-42.

VERNBERG, F. J., 1959. Studies on the physiological variation between tropical and temperate zone fiddler crabs of the Genus Uca. Biol. Bull. Woods Hole 117, 582-593.

WEBB, H. M. \& Brown, F. A. jr., 1961. Season variations in $\mathrm{O}_{2}$-consumption of $U c a$ pugnax. Biol. Bull. Woods Hole 121, 561-571.

Werner, B., 1954. Eine Beobachtung über die Winterwanderung von Arenicola marina. Helgol. Wiss. Meeresunters. 5, 93-102.

- 1954. Uber die Winterwanderung von Arenicola marina. Helgol. Wiss. Meeresunters. 5, 353-378.

Wreser, W., 1960. Meeresölkologie. Fortscbr. Zool. 12, 336-378.

\section{Diskussion im Anschluß an den Vortrag KRüger.}

WELLS: Dr. KRÜGER is to be congratulated on his careful and valuable work. I would like to ask (1) to what extent do you suppose that the oxygen consumption measurements reflect an influence of the unnatural conditions in the respirometer on the worm's behaviour and (2) is there any connection between seasonal changes in respiration and the reproductive cycle?

KR ÜGER: (1) Die Einzelmessungen beanspruchen etwa 1/2 Stunde, die einzelnen Rhythmen kann man daher nicht erkennen. Bei den ersten Messungen hatte ich meine Würmer in U-förmigen Glasrohren untergebracht. Durch die schon von VAN DAM in solchen Rohren beobachteten langen Pausen in der Ventilation waren keine gleichmäßigen Werte zu erhalten, da das Prinzip der Messung erfordert, daß die Würmer kontinuierlich ventilierten. Daher habe ich dieses Prinzip aufgegeben und mit einfachen Glasbehältern gearbeitet, in denen die Würmer sich während der Messung befinden. Größenordnungsmäßig erhielten wir die gleichen Werte. Genaueres läßt sich aber nicht aussagen. Ideal wäre es, die Messungen im Biotop durchzuführen, was aber sehr schwierig sein dürfte. Vielleicht ist es möglich, entsprechende Messungen im Kunstbau durchzuführen, aber auch hier bestehen große technische Schwierigkeiten. (2) Einen Einfluß des Sexualzyklus konnte ich nicht erkennen. Die im Herbst durchgeführten Versuche erfolgten kurz vor Ablage der Geschlechtsprodukte, die anderen in Frühjahr, ehe Geschlechtsprodukte sichtbar waren. Die Entwicklung der Geschlechtsprodukte erfolgt sicher während eines langen Zeitraumes, so daß sie sich im Stoffwechsel nicht so scharf ausprägt, wie man vielleicht erwarten möchte.

PROSSER: How long were the worms kept at a given temperature for the measurement of Q10? Have you attempted temperature acclimation experiments?

KrüGER: Eine Akklimatisation an eine bestimmte Temperatur erfolgte nicht. Die Tiere verblieben vor der Messung stets 1 bis 2 Stunden in der Atmungstemperatur, ehe mit den Messungen begonnen wurde. Erst nach dieser Zeit kann man konstante Werte erwarten. Während der Eingewöhnungszeit haben wir nichr gemessen, daher kann ich über diese Zeit nichts aussagen. Die von uns angewandte Methode mit kontinuierlichem Durchstrom benötigt einige Zeit zur Einstellung des Gleichgewichts zwischen Sauerstoffzufuhr und Sauerstoff verbrauch. Daher sind nach meiner Ansicht Messungen vor dieser Zeit unzuverlässig.

HoHENDORF: In der Zusammenstellung, in der Sie den Sauerstoffverbrauch bei verschiedenen Temperaturen von mehreren Versuchsgruppen miteinander verglichen, haben Sie ja den $\mathrm{O}_{2}-$ 
Verbrauch von theoretisch $1 \mathrm{~g}$ schweren Tieren, den Sie als den Wert $b$ aus den berechneten Regressionsgeraden entnommen haben, in Beziehung gesetzt. Dabei erhielten Sie beispielsweise bei Herbsttieren zwischen $15^{\circ}$ und $25^{\circ} \mathrm{C}$ einen nahezu konstanten $\mathrm{O}_{2}$-Verbrauch. Konnten diese Ergebnisse auch durch die rein empirischen Versuchsdaten von schweren Exemplaren bestätigt werden?

KRÜGER: Die durch die Werte $b$ und $a$ in ihrer Lage charakterisierten Regressionsgeraden schließen natürlich auch die Meßwerte für die schweren Exemplare ein. Durch die Temperaturabhängigkeit von $\alpha$ verschieben sich die Werte für die großen Würmer etwas gegeneinander. Im Prinzip läßt sich aber auch bei ihnen ein Temperaturintervall mit relativer Konstanz finden.

Wieser: Ich möchte die Frage von Professor Prosser noch einmal präzisieren. Bezieht sich die scheinbare Unabhängigkeit des $\mathrm{QO}_{2}$ von der Temperatur auf Tiere, die akuten, also momentanen Temperaturveränderungen ausgesetzt waren - und welches Zeitintervall verstreicht zwischen den Messungen bei zwei verschiedenen Temperaturen? Ist es möglich, den Anteil der Aktivität an diesen "Unstetigkeiten" des $\mathrm{QO}_{2}$ bei Temperaturänderung abzuschätzen?

KRÜGER: Der Anteil der Aktivität läßt sich nicht erkennen. Hier wären gesonderte Versuche notwendig, welche die Aktivität zu messen gestatten. Es handelt sich bei meinen Versuchen um akute Temperatureinwirkungen, wenn man von der relativ kurzen Eingewöhnungszeit absieht. Das Intervall zwischen zwei Temperaturstufen betrug etwa 2 Stunden.

OckelmanN: Sind die verschiedenen $\alpha$-Werte (in der Fomel $\mathrm{Y}=\mathrm{b}$. $\mathrm{w}^{a}$ ) saisonmäßig bedingt?

KRÜGER: Über einen Saison-Einfluß auf den $\alpha$-Wert kann ich keine sicheren Angaben machen. Die Bestimmung von $a$ bei den Frühjahrsversuchen ist dadurch etwas unsicher, daß zu dieser Jahreszeit die ganz kleinen Würmer, die für die Bestimmung der Parameter wichtig sind, schon zu stark herangewachsen sind. Bei den sehr hohen und niederen Temperaturen ergaben sich sehr starke Streuungen, die auch zu unwahrscheinlichen Werten für $a$ führten.

OHLE: Haben Sie auch die Beziehung zwischen Körperoberfläche von Arenicola und Respirationsaktivität untersucht?

KRÜGER: Die besondere Form der Größen-Abhängigkeit findet in der Größe von $\alpha$ ihren Ausdruck. Für eine oberflächenproportionale Atrnung müssen wir für $\alpha$ den Wert von 0,667 erwarten. Das war bei den Frühjahrstieren bei $15^{\circ} \mathrm{C}$ der Fall. Für eine gewichtsproportionale Atmung würde $\alpha$ den Wert 1 annehmen. Die Mehrzahl der $\alpha$-Werte liegt zwischen diesen beiden Grenzwerten, wie bei vielen anderen Invertebraten und Vertebraten. 